\title{
Discovery, characterization and potential roles of a novel NF-YAx splice variant in human neuroblastoma
}

Lucia Cappabianca $^{1 \dagger}$, Antonietta Rosella Farina ${ }^{1 \dagger}$, Lucia Di Marcotullio ${ }^{2,3}$, Paola Infante $^{4}$, Daniele De Simone ${ }^{1}$, Michela Sebastiano ${ }^{1}$ and Andrew Reay Mackay ${ }^{1 *}$ (D)

\begin{abstract}
Background: Identification of novel cancer-associated splice variants is of potential diagnostic, prognostic and therapeutic importance. NF-Y transcription factor is comprised of NF-YA, NF-YB and NF-YC subunits, binds inverted CCAAT-boxes in $\approx 70 \%$ of gene promoters, regulates $>1000$ cancer-associated genes and proteins involved in proliferation, staminality, differentiation, apoptosis, metabolism and is subject to component alternative splicing. RTPCR evaluation of alternative NF-YA splicing in primary human neuroblastomas (NBs), led to discovery of a novel NF-YAx splice variant, also expressed during mouse embryo development and induced by doxorubicin in NB cells. Here, we report the discovery and characterisation of NF-YAX and discus its potential roles in NB.

Methods: NF-YAx CDNA was RT-PCR-cloned from a stage 3 NB (provided by the Italian Association of Haematology and Paediatric Oncology, Genova, IT), sequenced and expressed as a protein using standard methods and compared to known fully-spliced NF-YAl and exon B-skipped NF-YAs isoforms in: EMSAs for capacity to form NF-Y complexes; by co-transfection, co-immunoprecipitation and Western blotting for capacity to bind Sp1; by IF for localisation; in AO/EtBr cell-death and colony formation assays for relative cytotoxicity, and by siRNA knockdown, use of inhibitors and Western blotting for potential mechanisms of action. Stable SH-SY5Y transfectants of all three NF-YA isoforms were also propagated and compared by RT-PCR and Western blotting for differences in cell-death and stem cell (SC)-associated gene expression, in cell-death assays for sensitivity to doxorubicin and in in vitro proliferation, substrate-independent growth and in vivo tumour xenograft assays for differences in growth and tumourigenic capacity.
\end{abstract}

Results: NF-YAx was characterized as a novel variant with NF-YA exons B, D and partial F skipping, detected in 20\% of NF-YA positive NBs, was the exclusive isoform in a stage 3 NB, expressed in mouse stage E11.5-14 embryos and induced by doxorubicin in SH-SY5Y NB cells. The NF-YAx protein exhibited nuclear localisation, competed with other isoforms in CCAAT box-binding NF-Y complexes but, in contrast to other isoforms, did not bind Sp1. NF-YAx expression in neural-related progenitor and NB cells repressed Bmi1 expression, induced KIF1Bß expression and promoted KIF1B $\beta$ dependent necroptosis but in NB cells also selected tumourigenic, doxorubicin-resistant, CSC-like sub-populations, resistant to NF-YAx cytotoxicity.

(Continued on next page)

\footnotetext{
* Correspondence: andrewreay.mackay@univaq.it

t'Lucia Cappabianca and Antonietta Rosella Farina contributed equally to this work.

'Department of Applied Clinical and Biotechnological Sciences, University of

L'Aquila, Via Vetoio, Coppito 2, 67100 L'Aquila, Italy

Full list of author information is available at the end of the article
}

(c) The Author(s). 2019 Open Access This article is distributed under the terms of the Creative Commons Attribution 4.0 International License (http://creativecommons.org/licenses/by/4.0/), which permits unrestricted use, distribution, and reproduction in any medium, provided you give appropriate credit to the original author(s) and the source, provide a link to the Creative Commons license, and indicate if changes were made. The Creative Commons Public Domain Dedication waiver (http://creativecommons.org/publicdomain/zero/1.0/) applies to the data made available in this article, unless otherwise stated. 


\begin{abstract}
(Continued from previous page)
Conclusions: The discovery of NF-YAx in NBs, its expression in mouse embryos and induction by doxorubicin in NB cells, unveils a novel NF-YA splice mechanism and variant, regulated by and involved in development, genotoxic-stress and NB. NF-YAx substitution of other isoforms in NF-Y complexes and loss of capacity to bind Sp1, characterises this novel isoform as a functional modifier of NF-Y and its promotion of KIF1Bß-dependent neural-lineage progenitor and NB cell necroptosis, association with doxorubicin-induced necroptosis and expression in mouse embryos coinciding with KIF1Bß-dependent sympathetic neuroblast-culling, confirm a cytotoxic function and potential role in suppressing NB initiation. On the other hand, the in vitro selection of CSC-like NB subpopulations resistant to NF-YAx cytotoxicity not only helps to explain high-level exclusive NF-YAx expression in a stage 3 NB but also supports a role for NF-YAx in disease progression and identifies a potential doxorubicin-inducible mechanism for post-therapeutic relapse.
\end{abstract}

Keywords: NF-Y, NF-YA, Neuroblastoma, Alternative splicing, Necroptosis, KIF1Bß, Cancer stem cells, Genotoxic stress

\section{Background}

Alternative gene splicing is a fundamental physiological mechanism for the differential expression of proteins from the same gene coding sequence and is largely responsible for the increased proteomic complexity of higher organisms that cannot be explained by differences in individual gene numbers alone [1]. Aberrant alternative splicing has been reported in cancer and is promoted by stress within the tumour microenvironment, oncogenic viral infection, gene translocation and oncogene de-regulation of splice factor expression. Cancerassociated alternative splicing has been shown to inactivate onco-suppressors and activate oncogenes, making the identification of novel cancer-associated splice isoforms of potential diagnostic, prognostic and therapeutic importance [1-5].

The ubiquitous transcription factor NF-Y binds inverted CCAAT-boxes ( $5^{\prime}$-ATTGG-3') in $\approx 70 \%$ of gene promoters, recruits other transcription factors and proteins to promoters and regulates $>1000$ cancer-associated genes involved in proliferation, stemness, differentiation, apoptosis, miRNA expression and metabolism [6-9]. NF-Y also regulates chromatin de-condensation during mitotic bookmarking and post-mitotic transcriptional re-activation, and acts as a bi-directional histone-like transcription factor, switching promoter histone methyl-marks from positive to negative. NF-Y is necessary for development, its inactivation is embryonically lethal and alterations in NF-Y activity have wide-ranging effects on cell behaviour [6-10]. The involvement of NF-Y in cancer is underpinned by its role in proliferation, the presence of inverted CCAAT-boxes in the promoters of many cancer-associated genes and interaction with and regulation of cancer-related proteins, including cfos, wild type and mutant p53, p73, $\Delta \mathrm{Np} 63 \alpha$, p21, Ash2L, BRCA-1, cMyc, Sox9, lamin, ZHX1/2 and in particular the ubiquitous cancer-related transcription factor $\mathrm{Sp} 1$ [6-11].

NF-Y is a hetero-trimer composed of NF-YA, NF-YB and NF-YC subunits, all of which are required for DNA binding and transcriptional activity. NF-YB complexes with NF-YC prior to binding NF-YA, which confers
DNA binding and transcriptional activity to the NF-Y complex [6-8, 12-14]. The $29.44 \mathrm{~kb} N F-Y A$ gene localises to chromosome $6 \mathrm{p} 21$, is organized into 9 exons [15] and is predominantly expressed as a fully-spliced $42 \mathrm{kDa}, 347$ amino acid (aa) long-form NF-YA $l$ with glutamine-rich, S/ T-rich transactivation, subunit-interaction and DNAbinding domains or an alternative exon B-spliced $40 \mathrm{kDa}$, 318 aa short-form NF-YAs, deleted of glutamine-rich aa's 26-54 [15, 16]. Minor NF-YA isoforms also include a $3 \mathrm{bp}$ aa 27 deletion-variant, a 18 bp aa 548-565 deletion-variant and L2-L6 alternative splice variants deleted in glutaminerich and S/T domains, driven by nucleotide 79,154 and 548 deletions at $\mathrm{A} / \mathrm{B}, \mathrm{B} / \mathrm{C}$ and $\mathrm{E} / \mathrm{F}$ splice junctions $[15,17]$.

Alternative splicing of the NF-YA gene has been implicated in the regulation of cell staminality, differentiation, apoptosis and transformation. NF-YAs forms part of the stem cell (SC) transcriptional circuitry, predominates in embryonic SCs and is lost upon SC differentiation. In contrast, NF-YA $l$ promotes differentiation and loss of NF-YA expression induces senescence or apoptosis. Alternative NF-YAs splicing is promoted by the oncogenic polyomavirus SV40 and by $v$-ras oncogene and converts tumorsuppressing, differentiation-promoting NF-Y complexes predominated by NF-YA $l$ into tumor and CSC promoting complexes predominated by NF-YAs [8, 18-23].

Neuroblastomas (NB) are aggressive embryonic tumours of neural crest origin, derived from immature sympathetic neuroblasts [24]. These primitive tumours initiate under conditions that impair sympathetic neuroblast culling during development, reported to depend upon either loss of the $K I F 1 B$ gene associated with chromosome 1p36deletion, germline KIF1B mutations or Nmyc amplification [25-33]. NF-Y involvement in NB pathogenesis and progression, however, has received scant attention. In the few existing reports, NF-Y has been shown to be critical for expression of soluble guanyl cyclase in NB cells required for cGMP production and differentiation [34] and is involved in elevated glypican 3 expression in NBs [35]. NF-Y and Sp1 transcription factors combine to promote tetramethylpyrazine-induced neuronal differentiation of 
NB cells [36] and regulate expression of the $\alpha 3 \mathrm{Na}+, \mathrm{K}+$ ATPase subunit, essential for maintaining electrochemical gradients across cell membranes [37]. Suboptimal NF$\mathrm{Y}$ function in NB cells has also been implicated in de-regulating the matrix metalloproteinase and tissue inhibitor of metalloproteinase equilibrium, resulting in invasion [38] and increased expression of the NF-YA subunit has been reported to differentiate between aggressive stage $4 \mathrm{NBs}$ and stage $4 \mathrm{~S} \mathrm{NBs}$ that exhibit spontaneous regression [39].

Considering the relative absence of studies of NF-Y expression in NB, combined with reports associating fully spliced NF-YA $l$ with cellular differentiation and reduced malignancy and associating alternative exon $\mathrm{B}$ spliced NF-YAs with cellular staminality and increased malignancy [18-23], we initiated a study of NF-YA $l$ and NF-YAs expression in human primary NBs. This led to the unexpected discovery of a novel NF-YAx splice variant, with NF-Y functional modifying activity, which forms the subject of this report.

\section{Materials and methods}

Aim, design and setting

The aim of this study was to report the discovery and characterization of the novel alternative NF-YAx splice variant, discovered as the exclusive NF-YA isoform expressed in an advanced stage $3 \mathrm{NB}$, expressed during mouse embryo development and induced in NB cells by doxorubicin, and to provide insights into its potential function in NB pathogenesis and progression. Experimental design included cloning and sequence characterization, expression vector construction, protein expression and characterization, transient and stable transfection and biological characterizations in terms of growth, cytotoxicity and tumorigenicity.

\section{Characteristics of participants and materials}

RNAs from primary human stage 1,2, 3 and 4 NBs (International NB Staging System) were kindly provided by the tissue bank of the Italian Association of Haematology and Pediatric Oncology (Genova, Italy). Patient age, sex or gender was not provided. RNAs from stage E8.5-E18.5 mouse embryos were provided by Dr. Rita Gallo (University of L'Aquila, L'Aquila, Italy). The following cell lines were used: stable transfected pcDNA3.1 control, TrkAIII and TrkT3 SH-SY5Y cell lines [3]; SV40 large T-antigen immortalized ST14A rat neuronal progenitor cells (kindly provided by Dr. Elena Cattaneo) [40]; NB cell lines SK-N-SH (HTB-11); SH-SY5Y (CRL2266); IMR32 (CCL-127); SK-N-BE (CRL-2271); LAN-1 (HB-8568), HEK-293 embryonic neural lineage kidney cells [41, 42] (CRL-1573) and SL2 Sneider's Drosphila Line 2 insect (CRL-1963) cell lines from ATCC (Rockville, MD); LAN-5 NB cell line from DSMZ (ACC673)
(Braunschweig, Germany), and SMS-KCNR and SH-EP $\mathrm{NB}$ cell lines from Dr. U.P. Thorgiersson (NCI, NIH, Bethesda, MD). Cells were routinely grown at $37^{\circ} \mathrm{C}$ and $5 \%$ $\mathrm{CO}_{2}$, in ATCC or DSMZ recommended culture medium (RPMI, DMEM), supplemented with appropriate antibiotics (Zeocin for stable-transfectants, penicillin and streptomycin) and $10 \%$ foetal calf serum. SL2 cells were cultured in Schneider's Drosophila medium supplemented with $10 \%$ foetal bovine serum. Human foetal brain-derived neural stem cells were maintained as neurospheres in NPMM maintenance medium supplemented with Growth and Supplement Singlequotes, as directed (Cambrex Bio Science, Milan, Italy). All cell lines were authenticated at source. The following antibodies were used: KIF1B $\beta$ (A301-055A-M, Bethyl Laboratories, Montgomery, TX); EglN3 (BS0532R-TR, Bioss, Woburn, MA); $\beta$-Actin (sc-47, 778), Caspase 9 (sc-819), Mcl1 (sc56077), Bcl2 (sc-509), NF-YA (sc-10,779), NF-YB (sc-13,045), Sp1 (sc-420), Ubiquitinated proteins (sc-9133, P62 (sc48402), Bcl-xL (sc136,207) (Santa Cruz, Dallas, TX); Alix (92880), Caspase 3 (9662), PARP (9642), pJNK (4668) and JNK (9258) (Cell Signaling Technology, Danvers, MA).

\section{RNA purification and RT-PCR}

Total RNAs from cell lines and xenograft tumor tissues were purified using an RNA-easy Plus RNA purification kit, as directed (Qiagen, Milan, Italy). All RNAs $(1 \mu \mathrm{g})$ were reverse transcribed using a Maxima $\mathrm{H}$ Minus First Strand cDNA synthesis kit, as directed (Thermo-Fisher Scientific, Waltham, MA) and subjected to semi-quantitative PCR (35 cycles of $40 \mathrm{~s}$ at $95^{\circ} \mathrm{C}, 30 \mathrm{~s}$ at $1^{\circ}$ below melting temperature and $42 \mathrm{~s}$ at $72{ }^{\circ} \mathrm{C}$ ), using the following primer sets: human NF-YA (5'-AATAGTTCGACAGA GCAGATTG-3' and 5'-TCCTGCCAAAC TGGCTG CTGGGAT-3', generating products of 575 bp for NFYAl, $488 \mathrm{bp}$ for NF-YAs and $338 \mathrm{bp}$ for NF-YAx); mouse NF-YA (5' -AAACAGCAATAGTTCCACAGAG CAGATCG-3' and 5'-CGATCTGCTCTGTGGAAC TATTGCTG TT T-3'; products of $582 \mathrm{bp}$ for NF-YAl, $494 \mathrm{bp}$ for NF-YAs and $361 \mathrm{bp}$ for NF-YAx); CD133 (5'-GCTGATGTTGAAACTGCTTGAG-3' and 5'-GG TGCCGTTGCCTTGG-3'); SOX2 (5'-CCAAGACGCT CATGAAGAAG-3' and 5'-TGGTCATGGAGTTGTACT GC-3'); CD117 (5'-AACGCTCGAGTACCTGTGAA-3' and 5'-GACAGAATTGATCCGCACAG-3'); Nestin (5'GGATCAGATGACATTAAGACCC-3' and 5'-TCCAGT GGTTCTTGAATTTCC-3'); Nanog (5'-TCTCTCCTCT TCCTTCCTCCATG-3' and 5'-CTGTTTGTAGCTGA GGTTCAGGATG-3'); $75^{\text {NGFR }}$ (5'-GTGGGACAGAGT CTGGGTGT-3' and 5'-AAGGAGGGGAGGTGATAGG A-3'); Bmil (5'-GCTGCCAATGGCTCTAATGA-3' and 5' -TGCTGGGCATCGTAAGTATCTT-3'); Bcl2 (5'-GAC T TCGCCGAGATGTCCAGC-3' and 5'-CAAGCT CCC ACCAGGGCCAAAC-3'); Mcl1 (5' -AAGCCAATGGGCA 
GGTCT-3' and 5' 'TGTCCAGTTTCCGAAGCAT-3'); BclxL (5'-GAACGGCGGCTGGGATACTTTTG-3' and 5'-GT GAATTCTGAGGCCAAGGGAAC-3'); PUMA (5'-GCATGGGGTCTGCCCAGG-3' and 5'-CCGCCG CTCGTACTGTGC-3'); BAD (5'-CAGTCACCAGCAGG AGCAG-3' and 5'-CCCTCCCTCCAAAGGAGACAG-3'); BAX (5'-ATGGACGGGTCCGGGGAGCAG-3' and 5'GCACCAGTTTGCTGGCAAAGTAG-3'); EglN3 (5'GCGTCTCCAAGCGACA-3' and 5'-GTCTTCAGTGAG GGCAGA-3'); KIF1B $\beta$ (5'-CAGTGACTGTAAGTTGTC TGATATA-3' and ' $5^{\prime}$-GTAAAGAGGCTCCTTGAAAT3'); GAPDH (5'-CTGCACCACCAACTGCTTAG-3' and 5'-AGGTCCACCACTGACACGTT-3') and 18S rRNA (5'-AAACGGCTACCA CATCCAAG-3' and 5' -CGCTCC CAAGATCCAACTAC-3').

\section{NF-YA variant CDNA cloning and sub-cloning}

NF-YA $x$ coding cDNA was cloned from the primary human stage $3 \mathrm{NB}$, exhibiting exclusive NF-YAx expression. NF-YAl, NF-YAs, NF-YB and NF-YC cDNAs were cloned from SH-SY5Y mRNA, using primers 5 -CCTCCTGATT GGGTTTCGGAGT-3' and 5'-GGGGTTAGGACACT CGGATGAT-3' spanning fully-spliced human NF-YAl cDNA (NM_002505.5); primers 5'-GGTTCTGACAGTA TTTCATGACAA-3' and 5'-CAGATCATGAAAACTGA ATTTGCT-3' spanning fully spliced human NF-YB cDNA (NM_006166) and primers 5'-GGACTCCTGA GCACAGTTGTCGAG-3' and $5^{\prime}$-CCTTGGCCTTGC CAGCTCAGGCCC-3' spanning fully spliced NF-YC cDNA (HSU78774). RT-PCR products were agarose gel purified, sub-cloned into the TA cloning vectors (Invitrogen, Thermo-Fisher Scientific, Waltham, MA) and sequenced in an ABI prism automated DNA-sequencer. Full length NF-YAl, NF-YAs, NF-YAx, NF-YB and NF-YC cDNA clones were then sub-cloned into mammalian pcDNA3.1Zeo $(+)$ and pAc insect expression vectors (Thermo-Fisher Scientific, Waltham, MA), together with cDNA for full-length Sp1, available in our laboratory. NFYAdn expression vector was from Dr. R. Mantovani (University of Milan, Italy).

\section{SiRNA knockdown}

SiRNA knockdown was achieved using a TriFECTa DicerSubstrate RNAi kit and three KIF1B-specific DicerSubstrate siRNA duplexes (hs.Ri.KIF1B.13.1: 5' -UCCACU GAGAAGGUCAGUAAAAUCA- 3 ' and $5{ }^{\prime}$-UGAUUUUA CUGACCUUCUCAGUGGAAA-3'; hs.Ri.KIF1B.13.2: 5' CAAGGAAUCCAAAUGCAUCAUUCAG-3' and $5^{\prime}-\mathrm{CU}$ GAAUGAUGCAUUUGGAUUCCUUGCU-3'; hs.Ri.KIF1 B.13.3: 5'-AGCUAUUGAACGUUUAAAG GAAUCA$\left.3^{\prime}\right)$, as directed (Integrated DNA Technologies, Bologna, Italy). Briefly, $1 \times 10^{5}$ cells $/ \mathrm{ml}$ HEK-293 cells on 24 well plates were grown overnight to $\approx 80 \%$ confluence and cotransfected with either $50 \mathrm{nM}$ of negative control siRNA duplex (5' -CGUUAAUCGCGUAUAAUACGCGUAT-3' and 5' -AUACGCGUAUUAUACGCGAUUAACGA C-3') or $50 \mathrm{nM}$ of a mix of KIF1B-specific siRNA duplexes, plus $1 \mu \mathrm{g} / \mathrm{ml}$ of pcDNA3.1 NF-YAx expression plasmid or empty pcDNA control plasmid, using TransIT-X2 Dynamic Delivery System, as directed (Mirus Bio, www. mirusbio.com/6000). Sham-transfected controls received transfection reagent alone. Knockdown of NFYAx-induced KIF1B $\beta$ expression was confirmed at 24 $h$ by RT-PCR and the effect of knockdown assessed on NF-YAx-induced cell death at $48 \mathrm{~h}$, by phase contrast and $\mathrm{AO} / \mathrm{EtBr}$ cell death assays. Knockdown experiments were performed in duplicate and repeated $(n=4)$. Transfection efficiency was confirmed using HPRT-S1 DS positive control and validated using a negative control duplex (NC1), as directed (Integrated DNA Technologies, Bologna, Italy).

\section{Immunoprecipitations and Western blotting}

Cell proteins, extracted in lysis buffer (PBS containing $0.5 \%$ sodium deoxycholate, $1 \%$ NP40, 0.1\% SDS, $1 \mathrm{mM}$ sodium orthovanadate, $1 \mathrm{mM}$ PMSF, $1 \mu \mathrm{g} / \mathrm{ml}$ of pepstatin A and Aprotinin), were analysed by reducing SDSPAGE/Western blotting, as previously described [3]. For immunoprecipitations, cell extracts $(200-500 \mu \mathrm{g})$, precleared with IgG and Protein A Sepharose (Fast flow, Sigma-Aldrich, St Louis, MI), were incubated overnight with primary antibody $(0.1-1.0 \mu \mathrm{g})$, with rotation at $4{ }^{\circ} \mathrm{C}$, then $20 \mu \mathrm{l}$ of Protein A Sepharose in lysis buffer was added and incubated for $30 \mathrm{~min}$ at $4{ }^{\circ} \mathrm{C}$. Protein A Sepharose/IgG conjugates, collected by centrifugation $(10,000$ $\mathrm{x} g$ for $5 \mathrm{~min}$ ), were washed in lysis buffer and examined by reducing SDS-PAGE/Western blotting.

\section{Stable SH-SY5Y transfection}

SH-SY5Y cells were stable-transfected with pcDNA 3.1 NF-YAl, NF-YAs or NF-YA $x$ or empty pcDNA3.1Zeo expression vectors, as previously described [3]. Zeocinresistant colonies were isolated upon appearance, clonally expanded and characterized by RT-PCR and Western blot.

\section{Transient transfections}

Cells at $1 \times 10^{5} / \mathrm{ml}$ were grown overnight to $\approx 80 \%$ confluence on 6 or 24 well plates, transfected with NF-YA variant or empty plasmid DNA $(1 \mu \mathrm{g} / \mathrm{ml})$ in Fugene HD, as directed (Promega, Madison, WI), washed at $6 \mathrm{~h}$, grown in complete medium, monitored at $6,12,24$ and $48 \mathrm{~h}$ for evidence of cytotoxicity and photographed. At $48 \mathrm{~h}$, suspension and adherent cell populations were separated and either analysed in $\mathrm{AO} / \mathrm{EtBr}$ cell-death assay, used for RNA purification and RT-PCR analysis or extracted for Western blotting. Transfection efficiency was estimated using pEGFP-N1 green fluorescent protein reporter plasmid (Clontech, Mountain View, CA). 


\section{a

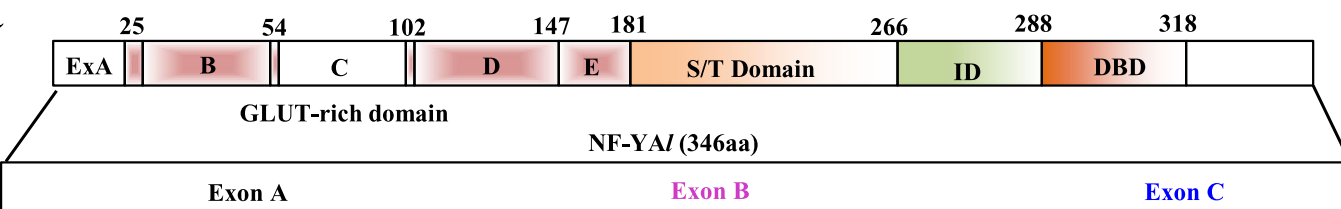 MEQYTANSNSSTEQIVVQAGQIQQQQGGVTAVQLQTEAQVASASGQQVQTLQVVQGQPLMVQVSGGQLITST Exon D GQPIMVQAVPGGQGQTIMQVPVSGTQGLQQIQLVPPGQIQIQGGQAVQVQGQQGQTQQIIIQQPQTAVTAGQTQ \\ Exon E
TQQQIAVQGQQVAQTAEGQTIVYQVPVNADGTILQQVTVPVSGMITIPAASLAGAQIVQTGANTNTTSSGQGTVT \\ Exon E
TQQQIAVQGQQVAQTAEGQTIVYQVPVNADGTILQQVTVPVSGMITIPAASLAGAQIVQTGANTNTTSSGQGTVT VTLPVAGNVVNSGGMVMMVPGAGSVPAIQRIPLPGAEMLEEEPLYVNAKQYHRILKRRQARAKLEAEGKIPKER RKYLHESRHRHAMARKRGEGGRFFSPKEKDSPHMQDPNQADEEAMTQIIRVS}

\section{NF-YAx (273aa)}

MEQYTANSNSSTEQIVVQAGQIQQQVQGQPLMVQVSGGQLITSTGQPIMVQAVPGGQ GQTIMQVPVSGTQGLQQTQQQIAVQGQQVAQTAEGQTIVYQVPVNADGTILQSGMITI PAASLAGAQIVQTGANTNTTSSGQGTVTVTLPVAGNVVNSGGMVMMVPGAGSVPAIQ RIPLPGAEMLEEEPLYVNAKQYHRILKRRQARAKLEAEGKIPKERRKYLHESRHRHA MARKRGEGGRFFSPKEKDSPHMQDPNQADEEAMTQIIRVS

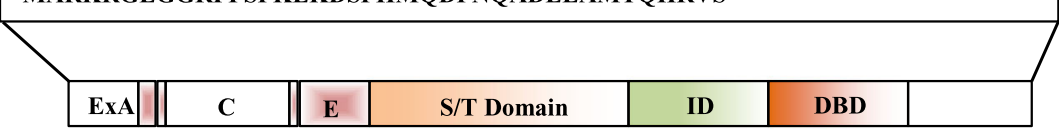

GLUT-rich domain

b

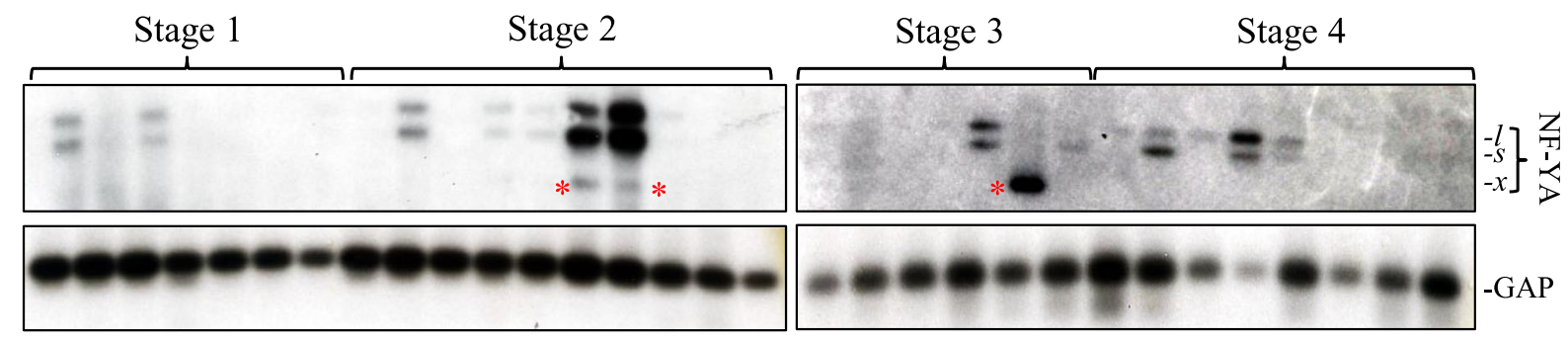

c
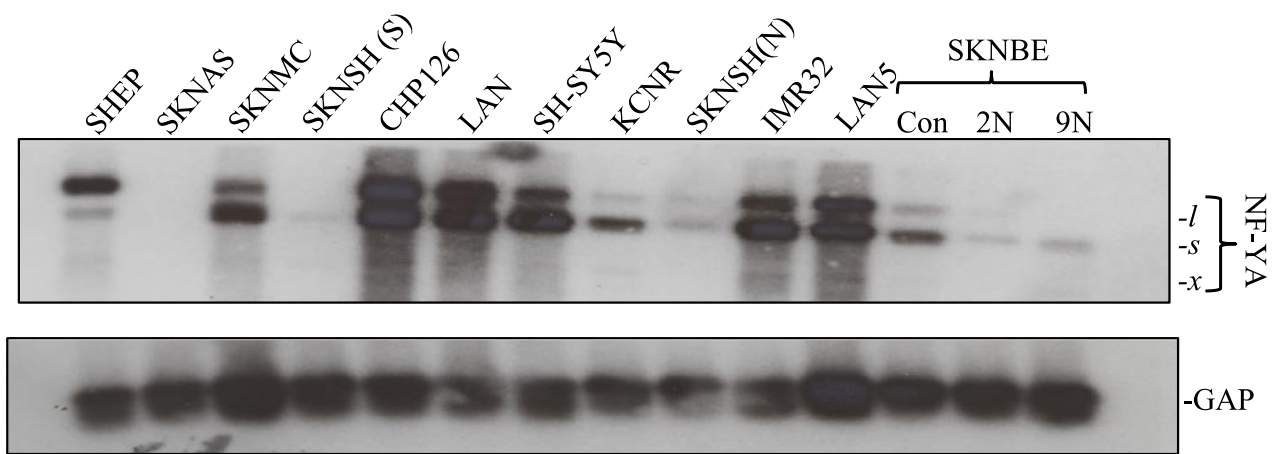

-GAP

d

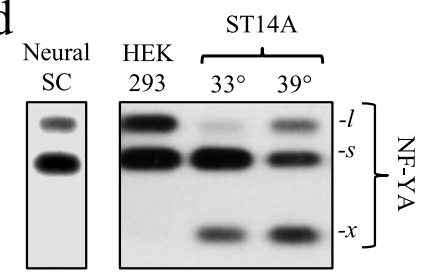

e

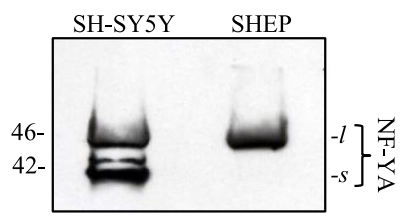

f

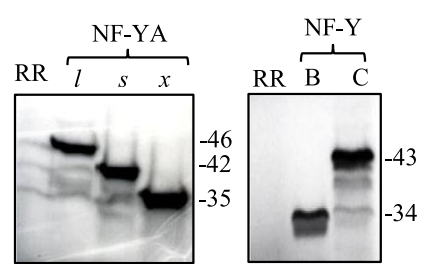

Fig. 1 (See legend on next page.) 
(See figure on previous page.)

Fig. 1 Neuroblastomas express NF-YAx. a Illustration of NF-YA glutamine-rich transactivation, serine threonine-rich (S/T), interaction (ID) and DNA binding (DBD) domains with relative positions and amino acid sequences of exons B and D, in NF-YAl and NF-YAx isoforms. Southern blots showing NF-YA isoform and GAP RT-PCR levels in: $\mathbf{b}$ primary stage 1-4 human NBs; c NB cell lines and human embryonic neural SC (left panel) and $\mathbf{d}$ HEK-293 and ST14A cells (right panel), hybridized with NF-YA exon C (upper panels) and GAP (lower panels) probes (* indicates NF-YAx). e Western blot showing NF-YAl and NF-YAs protein levels in SH-SY5Y and SHEP extracts (30 $\mu \mathrm{g})$. $\mathbf{f}$ Autoradiographs demonstrating in vitro translated NF-YAl, NF-YAs, NF-YAx (left panel), NF-YB and NF-YC proteins (right panel)

\section{Southern blotting}

Southern blots of NF-YA and GAPDH RT-PCR products were prepared, as previously described [3]. Briefly, RTPCR products from primary human NB RNAs $(1 \mu \mathrm{g})$ and doxorubicin-treated SH-SY5Y mRNAs $(100 \mathrm{ng})$, resolved by $1.5 \%$ agarose gel electrophoresis, were in-gel denatured with $0.5 \mathrm{M} \mathrm{NaOH}$, transferred to Hybond $\mathrm{N}+$ nylon membranes (Amersham International, Little Chalfont, UK) and hybridized with $\mathrm{P}^{32}$-labelled probes for either NF-YA exon C (5' -CAAGGGCAGCCATTAATGGTGCAGGTCAGT GGAGGCCAGCTAATCACATCAACTGGCCAACCCA TCATG-3'), NF-YA exon D (5'-AGGGCCAGCAGGGC CAGACCCAGCAGATCATCATCCAGCAGCCCCAG ACGGCT GTCACTGCTGGCCAGA-3') or GAPDH and visualized by autoradiography.

\section{MTS proliferation and thymidine incorporation assays} In MTS proliferation assays (Promega, Madison, WI), $5 \times 10^{3}$ cells in 96-well microtiter plates were grown for $0-4$ days. MTS reagent $(20 \mu \mathrm{l})$ was added at 24 -h intervals to individual wells and colorimetric conversion monitored at $492 \mathrm{~nm}$ every $30 \mathrm{~min}$ for $4 \mathrm{~h}$ in a microtiter plate reader. Assays were performed in duplicate and repeated three times $(n=6)$. For thymidine incorporation assays, $5 \times 10^{3}$ cells seeded on 96-well microtiter plates were grown for 0-4 days. At 24-h intervals, $1 \mu \mathrm{Ci}$ of tritiated $\left[{ }^{3} \mathrm{H}\right]$ thymidine (Amersham International, Little Chalfont, UK) was added and incubated for $4 \mathrm{~h}$. Cultures were then washed in pre-warmed PBS, trypsinized, vacuum transferred onto 3MM Whatman paper filters and counted in a $\beta$-Scintillation counter (Becton, Dickinson and Company, Franklyn Lakes, NJ). Assays were performed in duplicate and repeated 3 times $(n=6)$.

\section{In vitro protein translation}

NF-YA $l$, NF-YAs, NF-YA $x$, NF-YB and NF-YC proteins were translated in vitro from linearized cDNAs $(1 \mu \mathrm{g})$, in the presence of ${ }^{35} \mathrm{~S}$ labelled methionine, for $90 \mathrm{~min}$ at $37^{\circ} \mathrm{C}$, in a rabbit reticulocyte $\mathrm{T}_{\mathrm{N}} \mathrm{T}$ T7 Quick Coupled Transcription/translation System, as directed (Promega, Madison, WI). Translated proteins were resolved by reducing SDS-PAGE autoradiography. For EMSAs, ${ }^{35} \mathrm{~S}$-labelled methionine was omitted from in vitro translation reactions.

\section{In vitro Colony formation assays}

Cell suspensions $\left(1 \times 10^{5} / \mathrm{ml}\right)$ in 6 well culture plates were grown overnight to $\approx 80 \%$ confluence, transfected with empty pcDNA3.1 or pcDNA3.1 NF-YAl, NF-YAs or NFYAx expression vectors $(2 \mu \mathrm{g} / \mathrm{ml})$ in Fugene, as directed (Promega, Madison, WI), and grown for a further $48 \mathrm{~h}$. Zeocin $(200 \mu \mathrm{g} / \mathrm{ml})$ was then added, replaced every 3 days and colonies grown for 21 days, fixed in 100\% Methanol, stained in $0.5 \% \mathrm{w} / \mathrm{v}$ crystal violet, $1 \%$ Formaldehyde, $1 \mathrm{x}$ PBS, 1\% methanol, washed in tap water, air dried, photographed and counted. Assays were performed in triplicate and repeated 3 times $(n=9)$.

\section{Neural stem cell sphere growth assays}

Cells seeded at $1 \times 10^{5} / \mathrm{ml}$ in vertical T75 culture flasks (NUNC) were grown in DMEM/F1, containing $0.6 \%$ glucose, 1 x B27 supplement (Gibco, Thermo-Fisher Scientific, Waltham, MA), 1 x N2 supplement (Gibco), antibiotics (1 x Pen/Strep), glutamine, EGF (40 ng/ml) and FGF $(40 \mathrm{ng} / \mathrm{ml})$, and monitored for formation of neurospheres consisting of $>50$ cells. Assays were performed in duplicate and repeated $(n=4)$.

\section{Direct RT-PCR sequencing}

Agarose gel-purified RT-PCR product sequencing was performed using BigDye Direct Cycle Sequencing kit, as directed (Thermo-Fisher Scientific, Waltham, MA). Briefly, cDNAs (final concentration $4 \mathrm{ng} / \mathrm{ml}$ ) were added to reaction mixtures containing forward or reverse primer $(0.8 \mu \mathrm{M})$, BigDye direct PCR master mix, deionized water in a final volume of $20 \mu \mathrm{l}$ and subjected to 35 PCR cycles $\left(94^{\circ} \mathrm{C}\right.$ and for $10 \mathrm{~min}$ and $96^{\circ} \mathrm{C}$ for $10 \mathrm{~s}, 55^{\circ} \mathrm{C}$ for $5 \mathrm{~min}$ and $60^{\circ} \mathrm{C}$ for $4 \mathrm{~min}$ ). PCR reactions were mixed with $2 \mathrm{ul}$ of $\mathrm{NaAc}$ and $50 \mu \mathrm{l}$ absolute ethanol, incubated for $10 \mathrm{mins}$, spin-dried at $120000 \mathrm{rpm}$ for $20 \mathrm{mins}$ and sequenced in a mono-capillary sequencer, as directed (ABI PRISM 310, Life Technologies, Monza, IT).

\section{Tumor growth in soft agar}

Single-cell suspensions (passed through a gauge $\times 18$ syringe needle) of $5 \times 10^{4}$ cells were mixed in a $33 \%$ solution of agar (BiTec; Difco) in RPMI containing 5\% FCS at $37{ }^{\circ} \mathrm{C}$ and layered onto a solid $0.6 \%$ agarose substrate prepared in the same growth medium. Following agar solidification, complete medium was added, replaced 


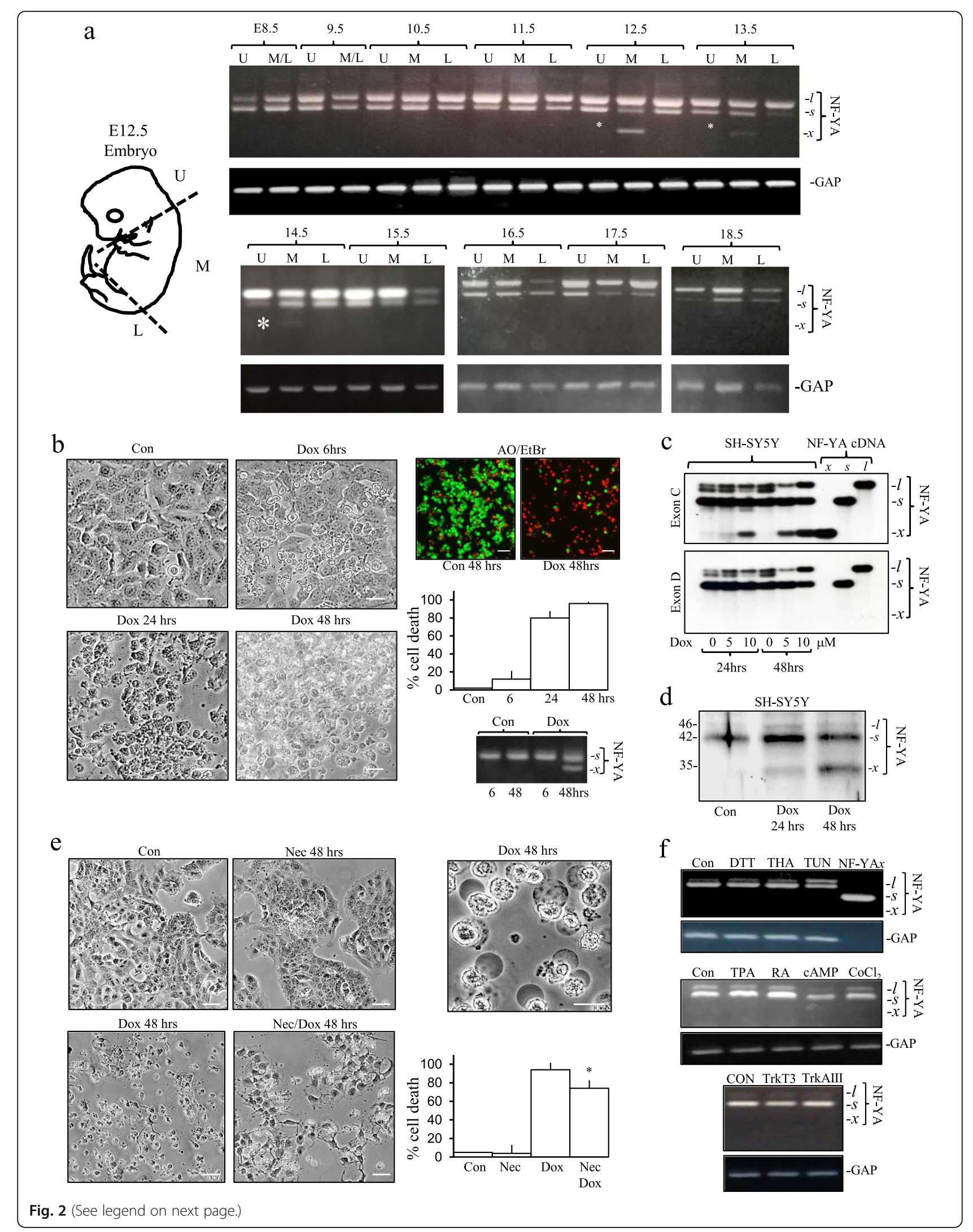


(See figure on previous page.)

Fig. 2 NF-YAx is development-regulated and induced by doxorubicin in neuroblastoma cells. a Mouse embryo upper (U), mid (M) and lower (L) regions used for RT PCR plus representative agarose gels demonstrating NF-YA isoform (upper panels) and GAP (lower panels) RT-PCR levels in stage E8.5 - E18.5 mouse embryo U, M and L regions (* indicates NF-YAx). b Representative micrographs (bar $=50 \mu \mathrm{m})$ of SH-SY5Y death induced by doxorubicin $(10 \mu \mathrm{M})$ at 6,24 and $48 \mathrm{~h}$, a representative AO/EtBr assay demonstrating dead (red) and living (green) SH-SY5Y cells following incubation without (Con $48 \mathrm{~h}$ ) or with $10 \mu \mathrm{M}$ doxorubicin (Dox $48 \mathrm{~h}$ ) (bar $=100 \mu \mathrm{m}$ ) plus a histogram demonstrating time-dependent SH-SY5Y cell death induced by $10 \mu \mathrm{M}$ doxorubicin at 6, 24 and $48 \mathrm{~h}$, displayed as mean ( \pm SD) percent death in three independent assays, performed in duplicate, and RT-PCR demonstrating the induction of NF-YAx expression in SH-SY5Y cells, following 48 treatment with $10 \mu \mathrm{M}$ doxorubicin (Dox) compared to untreated controls (Con). c RT-PCR Southern blots demonstrating time and concentration-dependent doxorubicin induction of NFYAx expression in SH-SY5Y cells together with NF-YA isoform CDNA standards (NF-YA CDNA), in blots hybridized with NF-YA exon C probe (Exon C, upper panel) but not with NF-YA exon D probe (Exon D, lower panel). d Western blot demonstrating doxorubicin (10 $\mu \mathrm{M}$ for 24 and $48 \mathrm{~h}$ ) induction of endogenous NF-YAx protein expression in SH-SY5Y nuclear extracts (50 $\mu \mathrm{g})$. e) Representative micrographs of SH-SY5Y cells untreated (Con) or treated for 48-h with $100 \mu \mathrm{M}$ necrostatin- 1 (Nec), $10 \mu \mathrm{M}$ doxorubicin (Dox) or with $100 \mathrm{mM}$ necrostatin-1 plus $10 \mathrm{mM}$ doxorubicin (Nec/Dox) and micrograph demonstrating cellular swelling, vacuolization and lysis of doxorubicin-treated (10 $\mu \mathrm{M}$ for $48 \mathrm{~h}$ ) SH-SY5Y cells $\left(\mathrm{bar}=50 \mu \mathrm{m}\right.$ ) plus a histogram demonstrating significant inhibition $\left(^{*}\right)$ of SH-SY5Y cell-death induced by $10 \mu \mathrm{M}$ doxorubicin (Dox) in the presence of $100 \mu \mathrm{M}$ necrostatin-1 (Nec/Dox) at $48 \mathrm{~h}$, compared to untreated controls and in the presence of $100 \mu \mathrm{M}$ necrostatin-1 (Nec) alone, displayed as mean ( \pm SD) percent death in three independent assays, performed in duplicate. $\mathbf{f}$ RT-PCRs of NF-YA isoform (upper panels) and GAP (lower panels) levels in SH-SY5Y cells treated for $48 \mathrm{~h}$ without (CON) or with TPA $(10 \mathrm{ng} / \mathrm{ml})$, retinoic acid (RA, $10 \mu \mathrm{M})$ ), dibutyryl CAMP (CAMP, 0.5 mM), CoCl $2(150 \mu \mathrm{M}), \mathrm{DTT}(5 \mathrm{mM})$, thapsigargin (THA, $10 \mathrm{ng} / \mathrm{ml})$ and tunicamycin (TUN, $1 \mu \mathrm{M})$ plus NF-YAx CDNA standard (NF-YAx) (upper left panel), and NF-YA isoform and GAP levels in stable TrkT3 and TrkAll SH-SY5Y-transfectants (bottom 2 panels).

every 2 days and growth monitored over 14 days. Assays were performed in duplicate and repeated $(n=4)$.

\section{Tumor growth in NGS mice}

Tumorigenesis in vivo was performed as previously described [3]. Stable-transfected SH-SY5Y cells, prepared as a single-cell suspension in PBS without $\mathrm{Ca}^{2+}$ and $\mathrm{Mg}^{2+}$, were injected subcutaneously into the flanks of anesthetized, 6-week-old female NGS mice (Charles River, Calco, Italy), at a concentration of $1 \times 10^{7}$ cells in $200 \mu \mathrm{l}$ per site. Tumor initiation was recorded at a minimum volume (tumor length $\times$ [tumor width $]^{2} \times 0.44$ ) of $12 \mathrm{~mm}^{3}$ and animals sacrificed at 21 days. Groups consisted of 5 animals and the assay performed once $(n=5)$. All experiments were performed in accordance with Italian national and Rome University guidelines.

\section{Nuclear extracts and EMSA}

Nuclear extracts and EMSAs were performed as previously described [3]. Briefly, binding reactions, performed at room temperature for $20 \mathrm{~min}$, contained ${ }^{32} \mathrm{P}-5^{\prime}$ end-labelled, double-stranded oligonucleotide probes, $2 \mu \mathrm{g}$ of polydeoxyinosinic-deoxy-cytidilic acid, $5 \mu \mathrm{g}$ of nuclear extract and additional competitor DNAs or antibodies as specified in the figure legends. The oligonucleotides used were as follows: inverted CCAAT-box: 5'-GGGAGACCGTACGT GATTGGTTAATCTCTT-3' and non-specific: 5'-GGTC GATAGGGAATTTACACGC-3' oligonucleotides. All oligonucleotides were double stranded, the complementary affinity strands are not indicated. Assays were repeated 3 times $(n=3)$.

\section{Cell death assays}

Cell death assays were as previously described [43]. Suspension or adherent cells, detached in ice cold PBS containing $1 \mathrm{mM}$ EDTA, were transferred into sterile tubes, pelleted at $1000 \mathrm{x}$ g at $4{ }^{\circ} \mathrm{C}$, washed in ice cold PBS, re-pelleted, re-suspended in $25 \mu \mathrm{l}$ of PBS containing $2 \mu \mathrm{l}$ of acridine orange/ethidium bromide solution $(100 \mu \mathrm{g} / \mathrm{ml}$ acridine orange and $100 \mu \mathrm{g} / \mathrm{ml}$ ethidium bromide in PBS), mounted onto glass slides, examined immediately under a Zeiss "Axioplan-2" fluorescence microscope, digitally photographed and dead (orange/red nuclei) and live cells (green nuclei) counted. Assays were performed in duplicate and repeated 3 times $(n=6)$.

\section{Indirect immunofluorescence}

Cells grown on Nunc glass chamber slides (Sigma-Aldrich, St Louis, MI) were washed in PBS, fixed and permeabilized in $100 \%$ ice cold methanol $\left(-20^{\circ} \mathrm{C}\right)$, incubated for $1 \mathrm{~h}$ in blocking solution (1\% bovine serum albumin in PBS- $0.03 \%$ TX100), incubated for $2 \mathrm{~h}$ with primary antibody diluted in blocking solution at room temperature, washed, incubated with secondary fluorochrome-conjugated antibody diluted in blocking solution, for $1 \mathrm{~h}$ at room temperature, mounted with VectaMount (Vector Laboratories, Berlingame, CA) and observed using a Zeiss Axioplan 2 fluorescence microscope with digital camera and Leica M500 Image Manager software.

\section{Statistical analysis}

Data were analysed by Student's t-test (https://www. graphpad.com/quickcalcs/ttest1.cfm) and statistical significance associated with probabilities of $\leq 0.05$.

\section{Results}

This study, originally designed to evaluate fully-spliced NF-YA $l$ and alternatively exon B spliced NF-YAs mRNA expression in primary human NBs, resulted in the unexpected discovery of a novel NF-YA $x$ splice variant. 


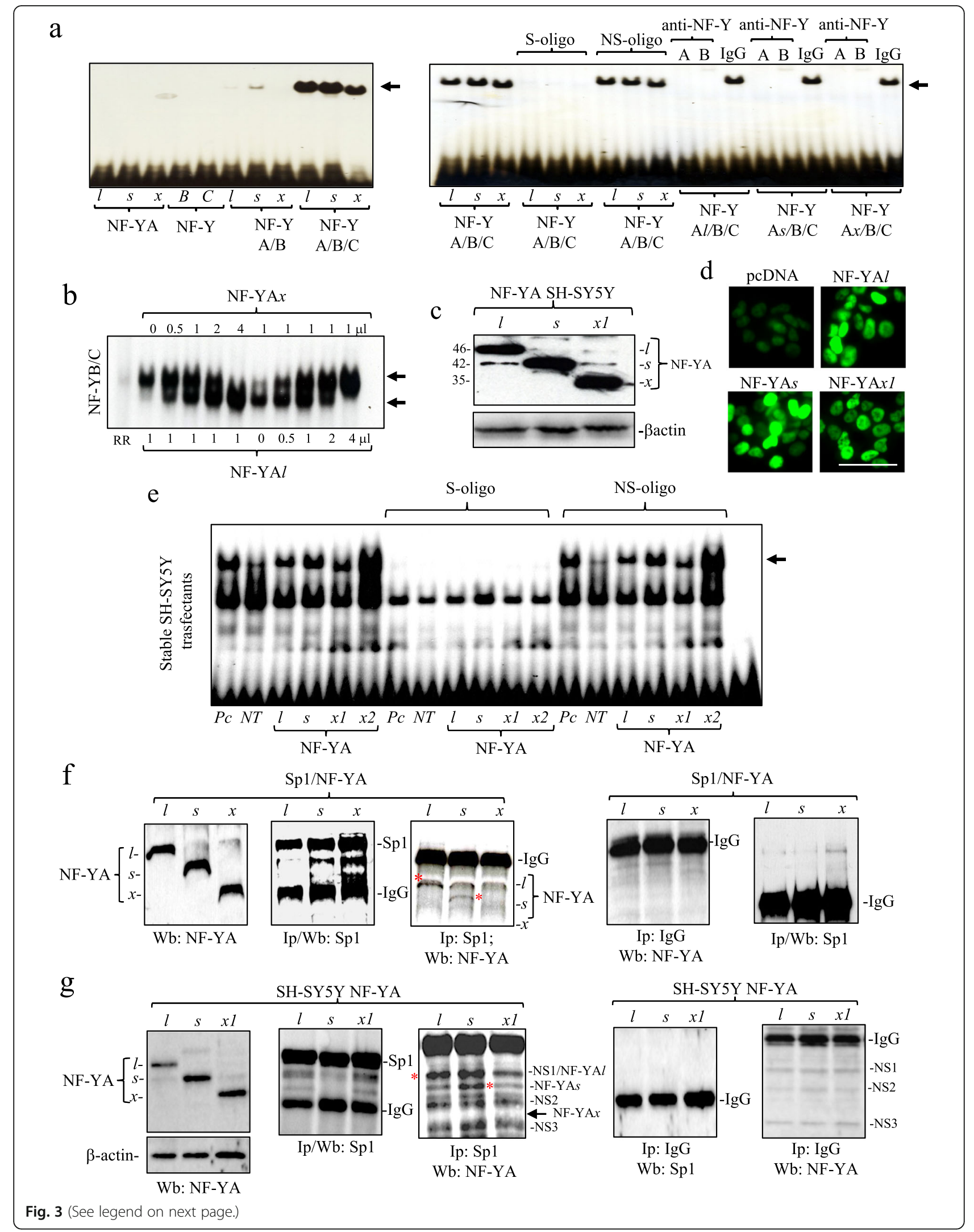


(See figure on previous page.)

Fig. 3 NF-YAx substitutes NF-YAl in NF-Y but does not bind Sp1. a EMSA demonstrating specific CCAAT-box binding NF-Y complexes (arrow) formed by in vitro translated NF-YAl, NF-YAs or NF-YAX, combined with NF-YB and NF-YC (left panel) plus competition EMSA showing inhibition of specific NF-Y binding complexes (arrow) in the presence of excess cold specific (S-oligo) but not non-specific (NS-oligo) oligonucleotide, and anti-NF-YB and anti-NF-YA antibodies but not pre-immune lgGs (lgG) (right panel). b EMSA showing changes in NF-Y electrophoretic mobility (arrows) under conditions of constant NF-YAl and increasing NF-YAx levels and vice versa, plus NF-YB and C, demonstrating reciprocal NF-YA/ and NF-YAx substitution in NF-Y complexes. c Western blot demonstrating NF-YAl, NF-YAs, NF-YAx and $\beta$-actin levels in stable NF-YAl, NF-YAs and NFYAX1 SH-SY5Y-transfectant extracts $(30 \mu \mathrm{g}$ ) (left panel) plus d IF demonstration of nuclear NF-YAl, NF-YAs and NF-YAx localization in stable NF-YAl, NF-YAs and NF-YAX1-SH-SY5Y transfectants (bar $=50 \mu \mathrm{m}$ ). e EMSA of specific CCAAT-box binding NF-Y complexes (arrow) in stable NF-YAl, NF-YAs, NF-YAX1 and NF-YAX2 SH-SY5Y-transfectant nuclear extracts, with competition EMSA demonstrating specificity by inhibition with cold specific (S) but not non-specific (NS) oligonucleotides. f IP/Western blots showing NF-YA isoform levels in SL2 extracts (30 $\mu \mathrm{g})$, $72 \mathrm{~h}$ following co-transfection with pAc SP1 and NF-YA insect expression vectors (1st panel); Sp1 and lgG levels in Sp1 immunoprecipitates (500 $\mu \mathrm{g}$ SL2 extracts) (2nd panel); Sp1 pull down of NF-YAl and NF-YAs (red asterisks) but not NF-YAx in Sp1 immunoprecipitates (500 $\mu \mathrm{g}$ SL2 extracts) (3rd panel), and the absence of Sp1, NF-YAl, NF-YAs and NF-YAx in pre-immune lgG precipitates (500 $\mu \mathrm{g}$ SL2 extracts) (4th and 5th panels). $\mathbf{g}$ IP/ Western blots of NF-YA isoform and $\beta$-actin protein levels in stable NF-YAl, NF-YAs and NF-YAx1 SH-SY5Y-transfectant extracts $(30 \mu \mathrm{mg})(1 \mathrm{st}$ panel); Sp1 and lgG levels in Sp1 immunoprecipiates (500 $\mu \mathrm{g}$ extracts) (2nd panel); Sp1 pulldown of NF-YAl and NF-YAs (*) (overlapping NS1 and NS2 bands) but not NF-YAx (arrow) (3rd panel), and the absence of Sp1, NF-YAl, NF-YAs and NF-YAx but not non-specific NS1, NS2 and NS3 bands in pre-immune immunoprecipitates (500 $\mu \mathrm{g}$ of each extract) (4th and 5 th panels)

\section{NF-YAx cloning and sequencing}

NF-YAl (1284 bp) and NF-YAs (1197 bp) coding cDNAs from SH-SY5Y cells and full-length NF-YAx cDNA $(947$ bp) from a stage $3 \mathrm{NB}$ were sequenced, and NF-YAx characterized as a novel splice variant exhibiting inframe exons B (87 bp, amino acids (aa) 26-54), D (132 bp, aa 103-146) and partial F (18 bp, aa 182-188) skipping (Fig. 1a) [15].

\section{NF-YAx is expressed in NBs and mouse embryos}

RT-PCR of NB RNAs using primers spanning NF-YA exons A-F, generated $575 \mathrm{bp}$ (NF-YAl), $488 \mathrm{bp}$ (NFYAs) and $338 \mathrm{bp}$ (NF-YAx) products that hybridized to NF-YA exon $C$ probe in Southern blots (Fig. 1b). The $338 \mathrm{bp}$ product, characterized as NF-YAx by direct PCR sequencing (not shown), was the exclusive isoform expressed in a stage $3 \mathrm{NB}$ and a minor isoform, together with NF-YA $l$ and NF-YAs, in two stage 2 NBs but was not detected in either stage 1 or 4 NBs. RNAs from SK-N-MC, SK-N-SH, SH-SY5Y, KCNR, IMR-32 and SK-N-BE NB cell lines, HEK293 embryonic neuronal-lineage kidney cells and human neonatal brain stem cells (SCs) exhibited predominant NF-YAs but not constitutive NF-YAx expression; CHP-126, LAN-1 and LAN-5 NB cell lines exhibited equivalent NF-YAs and NF-YA $l$ but not NF-YA $x$ expression and SHEP NB cells exhibited predominant NF-YA $l$ but not NF-YA $x$ expression. Predominant NF-YAs with minor NF-YAx expression characterized ST14A embryonic striatal neuronal progenitors under progenitor-maintaining $\left(33^{\circ} \mathrm{C}\right)$ and differentiation-inducing $\left(39^{\circ} \mathrm{C}\right)$ conditions [40] (Fig. 1c and d). Western blots confirmed predominant NFYAs protein expression in SH-SY5Y cells, predominant NF-YA $l$ expression in SHEP cell extracts (Fig. 1e). NF-YA isoforms translated in vitro were characterized by SDSPAGE with approximate molecular masses of $46 \mathrm{kDa}$ (NF$\mathrm{YAl}$ ), $42 \mathrm{kDa}$ (NF-YAs) and $35 \mathrm{kDa}$ (NF-YAx) (Fig. 1f).
Samples were not available for NF-YAx protein analysis in primary NBs.

In mouse embryo RNAs, NF-YAs mRNA expression was predominant in stage E8.5 embryos, NFYA $l$ mRNA expression predominant in stage E9.5E18.5 embryos and NF-YAx mRNA expression, confirmed by direct sequencing (data not shown), was detected in main body (M) but not head (U) or lower limbs (L) RNAs from stage E12.5, 13.5 and 14.5 embryos (Fig. 2a).

\section{Doxorubicin induces NF-YAx expression in SH-SY5Y cells}

Doxorubicin $(10 \mu \mathrm{M})$ induced NF-YA $x$ mRNA expression in SH-SY5Y cells at times of maximal >95\% cell death (Fig. 2b), which in Southern blots exhibited time and concentration-dependent induction kinetics at concentrations of 5 and $10 \mu \mathrm{M}$, with NF-YAx identified by hybridization to the NF-YA exon $C$ (Exon $C$ ) but not exon D (Exon D) probe (Fig. 2c). NF-YAx protein expression was also detected in SH-SY5Y nuclear extracts at 24 and $48 \mathrm{~h}$, following Doxorubicin $(10 \mu \mathrm{M})$ treatment (Fig. 2d). Necrostatin-1 (100 $\mu \mathrm{M})$ significantly reduced cell death induced by $10 \mu \mathrm{M}$ doxorubicin from $94 \pm 7.5 \%$ to $74 \pm 8.7 \% \quad(p<0.002, n=12)$, at $48 \mathrm{~h}, \quad($ Fig. $2 \mathrm{e})$ and doxorubicin-induced cell death was also characterised by vacuolation, swelling and lysis, consistent with a proportion of necroptotic cell-death (Fig. 2e). NF-YAx expression in SH-SY5Y cells was not induced by treatment with either DTT $(5 \mathrm{mM})$, thapsigargin $(10 \mathrm{ng} / \mathrm{ml})$, tunicamycin $(1 \mu \mathrm{M})$, TPA $(10 \mathrm{ng} / \mathrm{ml})$, dibutyryl cAMP $(0.5 \mathrm{mM})$, retinoic acid $(10 \mu \mathrm{M})$ or $\mathrm{CoCl}_{2}(150 \mu \mathrm{M})$ over $48 \mathrm{~h}$ and was not detected in either stable TrkT3 or TrkAIII SH-SY5Ytransfectants [3] (Fig. 2f).

\section{NF-YAx forms CCAAT-box binding NF-Y complexes}

In EMSAs, in vitro-translated NF-YAl, NF-YAs and NFYA $x$ all formed specific CCAAT-box binding complexes 
a

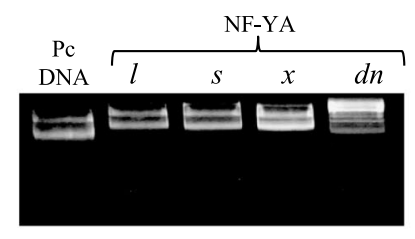

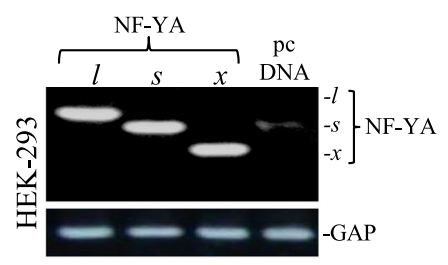

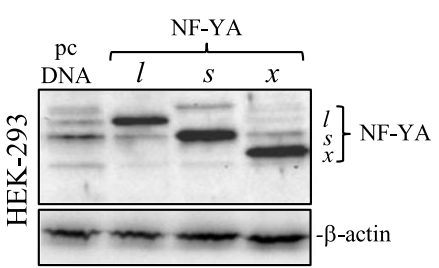

b
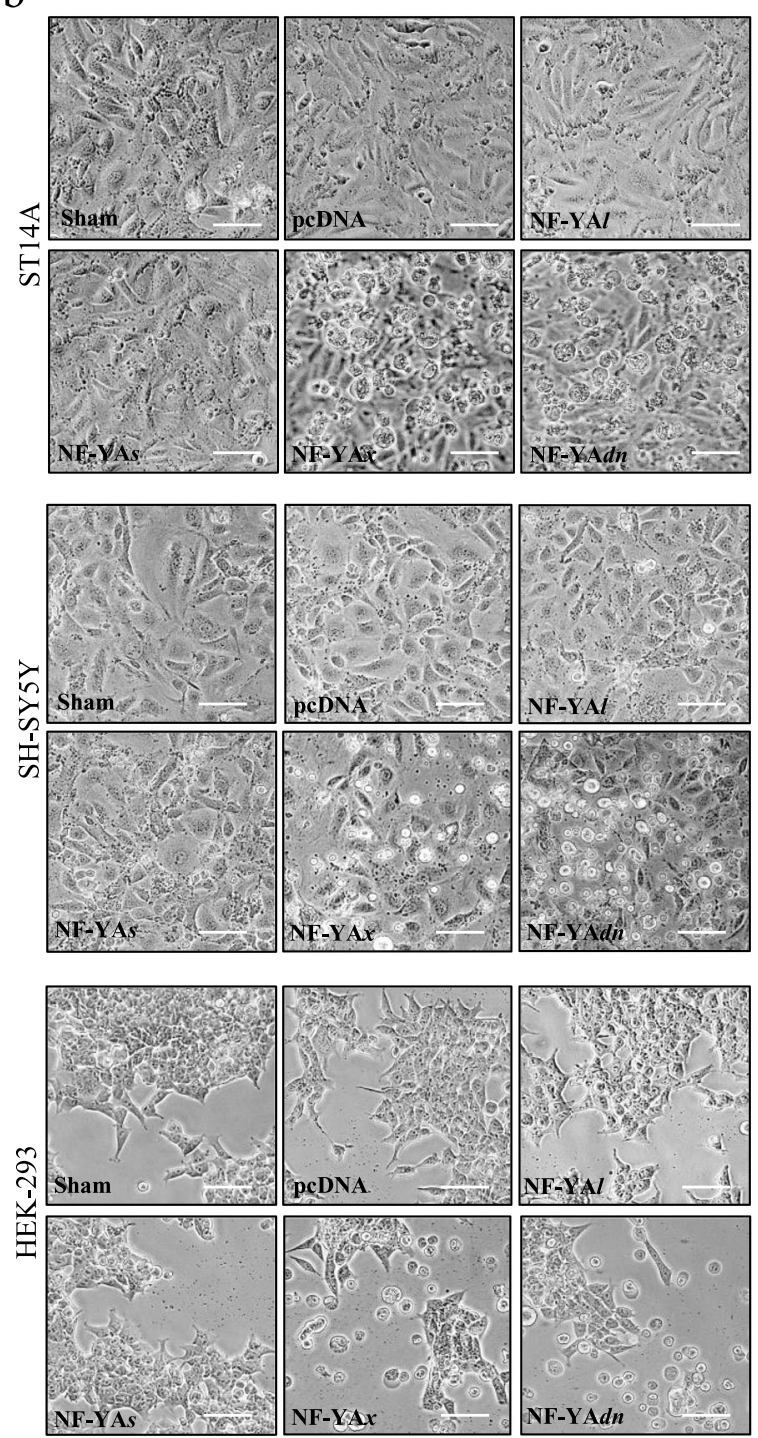

d

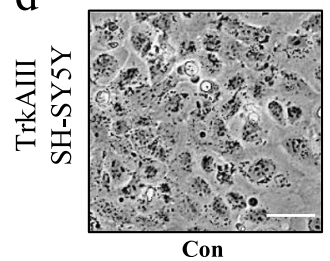

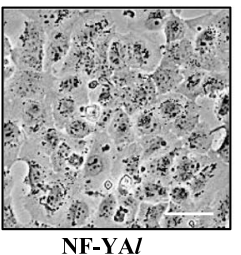

NF-YA $l$

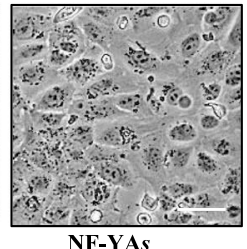

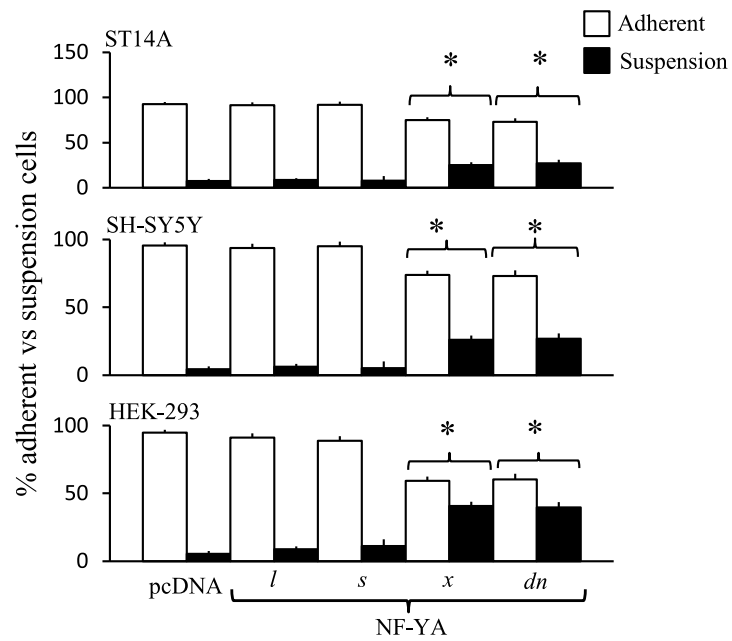
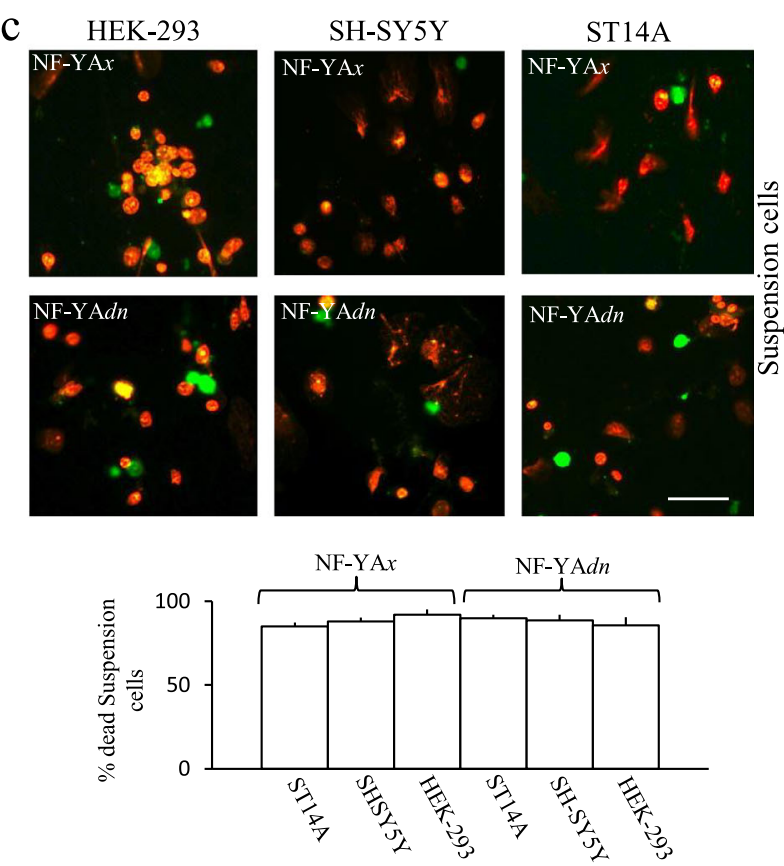

Fig. 4 (See legend on next page.)

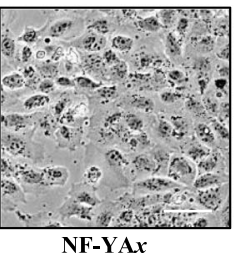

NF-YAx 
(See figure on previous page.)

Fig. 4 NF-YAx is cytotoxic. a Sterile purified pcDNA, NF-YAl, NF-YAs, NF-YAx and NF-YAdn plasmids used in transfections plus RT-PCR plus Western blots demonstrating NF-YAl, NF-YAs, NF-YAx and GAP mRNA and protein levels in HEK-293 cells, following $48 \mathrm{~h}$ transfection with pcDNA3.1 NFYAl, NF-YAs, NF-YAx and empty pcDNA mammalian expression vectors. b Representative Micrographs illustrating ST14A (upper 6 panels), SH-SY5Y (middle 6 panels) and HEK-293 (lower 6 panels) cell-death, following $48 \mathrm{~h}$ transfection with pcDNA, NF-YAl, NF-YAs, NF-YAx and NF-YAdn expression vectors $(\mathrm{bar}=100 \mu \mathrm{m})$ plus histograms demonstrating the percentage change in adherent and suspension (dead/dying) ST14A (upper), SH-SY5Y (middle) and HEK-293 (lower) cells, following $48 \mathrm{~h}$ transfection with pcDNA, NF-YAl, NF-YAs, NF-YAx and NF-YAdn expression vectors, results are displayed as mean $( \pm \mathrm{SD})$ percent in three independent assays performed in duplicate $\left({ }^{*}=\right.$ significant). $\mathbf{c}$ Representative $\mathrm{AO} /$ EtBr assays $(\mathrm{bar}=100 \mu \mathrm{m})$ demonstrating HEK-293, SH-SY5Y and ST14A suspension cell death (orange/red), $48 \mathrm{~h}$ following transfection with NFYAx and NF-YAdn expression vectors plus a histogram of the percentage of suspension cell-death, displayed as mean ( \pm SD) in three independent AO/EtBr assays performed in duplicate. $\mathbf{d}$ Micrographs demonstrating lack of stable TrkAlll transfected SH-SY5Y cell-death, following $48 \mathrm{~h}$ transfection with either NF-YAl, NF-YAs or NF-YAx expression vectors (bar $=100 \mu \mathrm{m})$

with NF-YB and NF-YC, confirmed by competition EMSAs in which excess un-labelled CCAAT-box oligonucleotide (S-oligo), anti-NF-YA and anti-NF-YB antibodies but not non-specific oligonucleotide (NS-oligo) or pre-immune IgG abrogated binding (Figs. If and 3a and b). EMSAs performed with constant NF-YAl, NF$\mathrm{YB}$ and NF-YC and increasing NF-YAx levels $(0-4 \mu \mathrm{l})$, or constant NF-YAx, NF-YB and NF-YC and increasing NF-YA $l$ levels $(0-4 \mu \mathrm{l})$, revealed reciprocal NF-YA $x /$ NFYAl substitution, confirmed by changes in electrophoretic mobility (Fig. 3b). EMSAs also detected specific CCAAT-box binding NF-Y complexes, containing NFYAl, NF-YAs and NF-YAx, in nuclear extracts from stable NF-YA isoform SH-SY5Y-transfectants, exhibiting similar levels of nuclear-localized NF-YAl, NF-YAs and NF-YA $x$ expression (Fig. 3c, d and e). NF-YAx-specific antibodies, however, are not available to confirm NFYA $x$ recruitment to gene promoters in vivo by chromatin immunoprecipitation assay.

\section{NF-YAx does not bind Sp1}

NF-YA $l$ and NF-YAs bind Sp1 and regulate Sp1 recruitment to promoters $[11,44]$. IP/Western blots confirmed Sp1 pull-down of NF-YA $l$ and NF-YAs but not NF-YA $x$ in Sp1-negative SL2 cells [45], co-transfected with pAc Sp1 and NF-YAl, NF-YAs and NF-YAx insect expression vectors (Fig. 3f). In stable SH-SY5Y-transfectants evidence for Sp1 pull down of NF-YA $l$ and NF-YAs but not NF-YA $x$ was also supported by augmented immunoreactivity overlapping non-specific (NS1 and NS2) bands, consistent with $\mathrm{Sp} 1$ binding of NF-YA $l$ and NF-YAs but not NF-YAx (Fig. 3g).

\section{$\mathrm{NF}-\mathrm{Y} x$ is cytotoxic to embryonal neural-lineage progenitors and NB cells and inhibits colony formation}

Transient 48-h transfection of ST14A, HEK-293 and SH-SY5Y with pEGFP-N1 reporter gene, resulted in transfection-efficiencies of $28.3 \pm 8.2 \%, 55.9 \pm 4.7 \%$ and $30.3 \pm 6.5 \%$ (mean $\pm \mathrm{SD})(n=6$, for all three), respectively (not shown). Transient 48-h ST14A, HEK-293 and SHSY5Y transfection with empty pcDNA, NF-YA $l$, NF-YAs,
NF-YA $x$ or NF-YAdn vectors, resulted in similar levels of NF-YA isoform expression (Fig. 4a, shown for HEK293 cells only).

In ST14A cells, NF-YAx and NF-YAdn transfection significantly increased the percentage of suspension cells at $48 \mathrm{~h}$, from $7.5 \pm 2.25 \%(n=6)$ in pcDNA, $8.5 \pm 2.1 \%(n=6)$ in NF-YA $l$ and $8 \pm 5.2 \%(\mathrm{n}=6)$ in NF-YAs-transfectants, to $25 \pm 3.1 \%(\mathrm{n}=6)$ in NF-YAx and $27 \pm 4.2 \%(\mathrm{n}=6)$ (means $\pm \mathrm{SD})$ in NF-YAdn-transfectants $(p<0.0001$ for both NFYA $x$ and NF-YAdn vs controls), with $98 \pm 5.5 \%(n=6)$ of NF-YA $x$-induced suspension cells and $95 \pm 7.6 \%(n=6)$ (means $\pm \mathrm{SD}$ ) of NF-YAdn-induced suspension cells confirmed dead by AO/EtBr assay (Fig. 4b and c). In SH-SY5Y cells, NF-YA $x$ and NF-YAdn also significantly increased the percentage of suspension cells at $48 \mathrm{~h}$, from $4.25 \pm 2.25 \%$ $(\mathrm{n}=6)$ in pcDNA, $6.25 \pm 2.1 \%(\mathrm{n}=6)$ in NF-YA $l$ and $5 \pm$ $5.2 \%(\mathrm{n}=6)$ in NF-YAs-transfectants, to $26 \pm 3.1 \%(\mathrm{n}=6)$ in NF-YAx and $26.75 \pm 4.2 \%(\mathrm{n}=6)$ in NF-YAdn-transfectants (means $\pm \mathrm{SD})(p<0.0001$ for NF-YAx and NF-YAdn vs controls), with $88 \pm 5.5 \%(\mathrm{n}=6)$ of NF-YAx-induced suspension cells and $93 \pm 5.6 \%(n=6)$ (means \pm SD) of NF$\mathrm{YAd} n$-induced suspension cells confirmed dead by $\mathrm{AO} /$ EtBr assay (Fig. 4b and c). In HEK-293 cells, NF-YAx and NF-YAd $n$ also significantly increased the percent of suspension cells, from $5.25 \pm 2.22 \%(\mathrm{n}=6)$ in pcDNA, $8.75 \pm 1.9 \%$ $(\mathrm{n}=6)$ in NF-YAl and $11.25 \pm 4.8 \%(\mathrm{n}=6)$ in NF-YAstransfectants, to $40.75 \pm 3.1 \%(\mathrm{n}=6)$ in NF-YA $x$ and $39.6 \pm$ $4.3 \%(\mathrm{n}=6)$ in NF-YAd $n$-transfectants (means $\pm \mathrm{SD})(p<$ 0.0001 for NF-YA $x$ and NF-YAdn vs controls), with $92 \pm$ $6.5 \%(n=6)$ of NF-YA $x$-induced suspension cells and $94 \pm$ $7.8 \%(n=6)($ means \pm SD) of NF-YAdn-induced suspension cells confirmed dead by $\mathrm{AO} / \mathrm{EtBr}$ assay (Fig. $4 \mathrm{~b}$ and c). In contrast, NF-YAl, NF-YAs or NF-YAx did not induce the death of SH-SY5Y cells expressing TrkAIII oncoprotein (Fig. 4d). NF-YAx-induced death was not detected prior to $16 \mathrm{~h}$, was maximal at $48 \mathrm{~h}$, was not prevented by $z$-VAD-fmk $(20 \mu \mathrm{M})$ but was significantly inhibited by necrostatin-1 $(100 \mu \mathrm{M})$ [46], from $29 \pm$ $3.1 \%(n=6)$ to $12 \pm 1.6 \%(n=6)$ in SH-SY5Y cells and from $30 \pm 4.5 \%(\mathrm{n}=6)$ to $15 \pm 1.5 \%(\mathrm{n}=6)$ in HEK293 cells (means \pm SD) $(P<0.001$, for both) (Fig. 5a). 
a

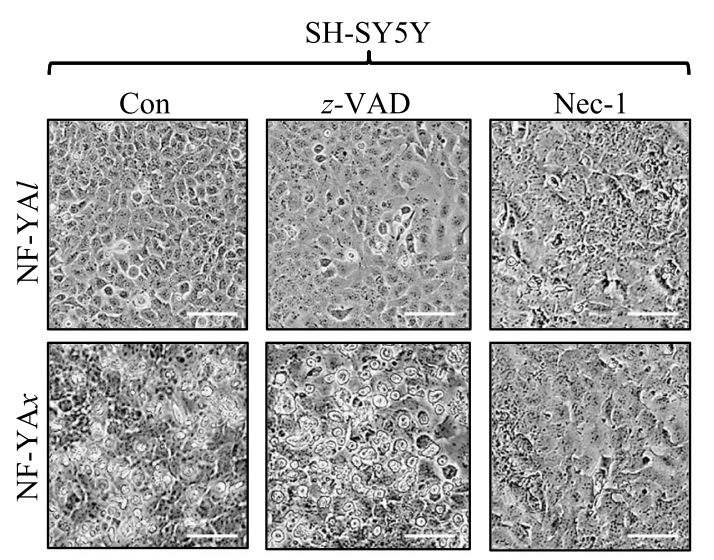

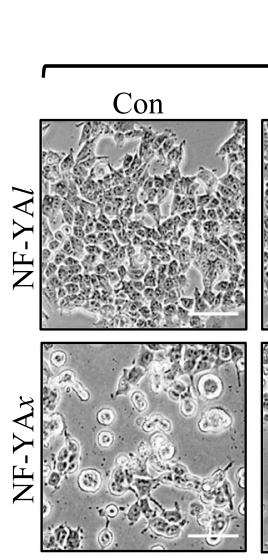

HEK293

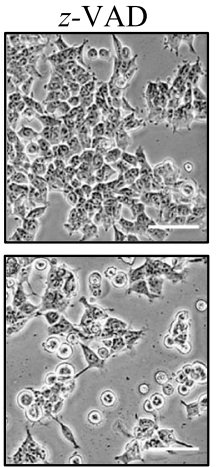

Nec-1
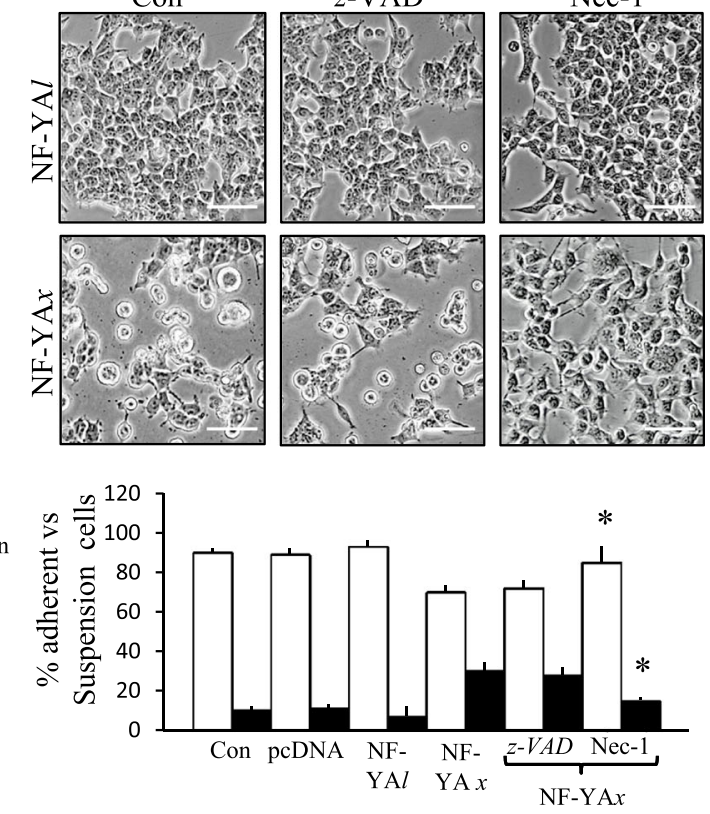

b

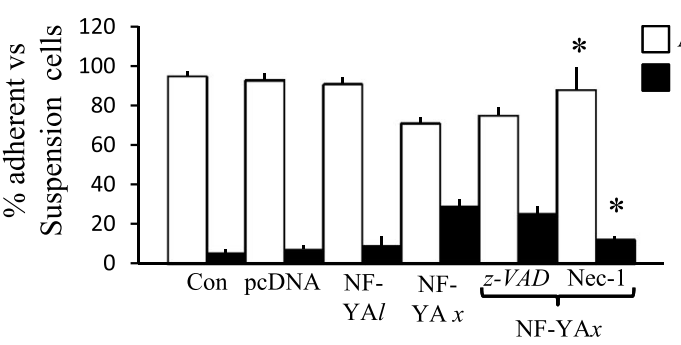

$\square$ Adherent
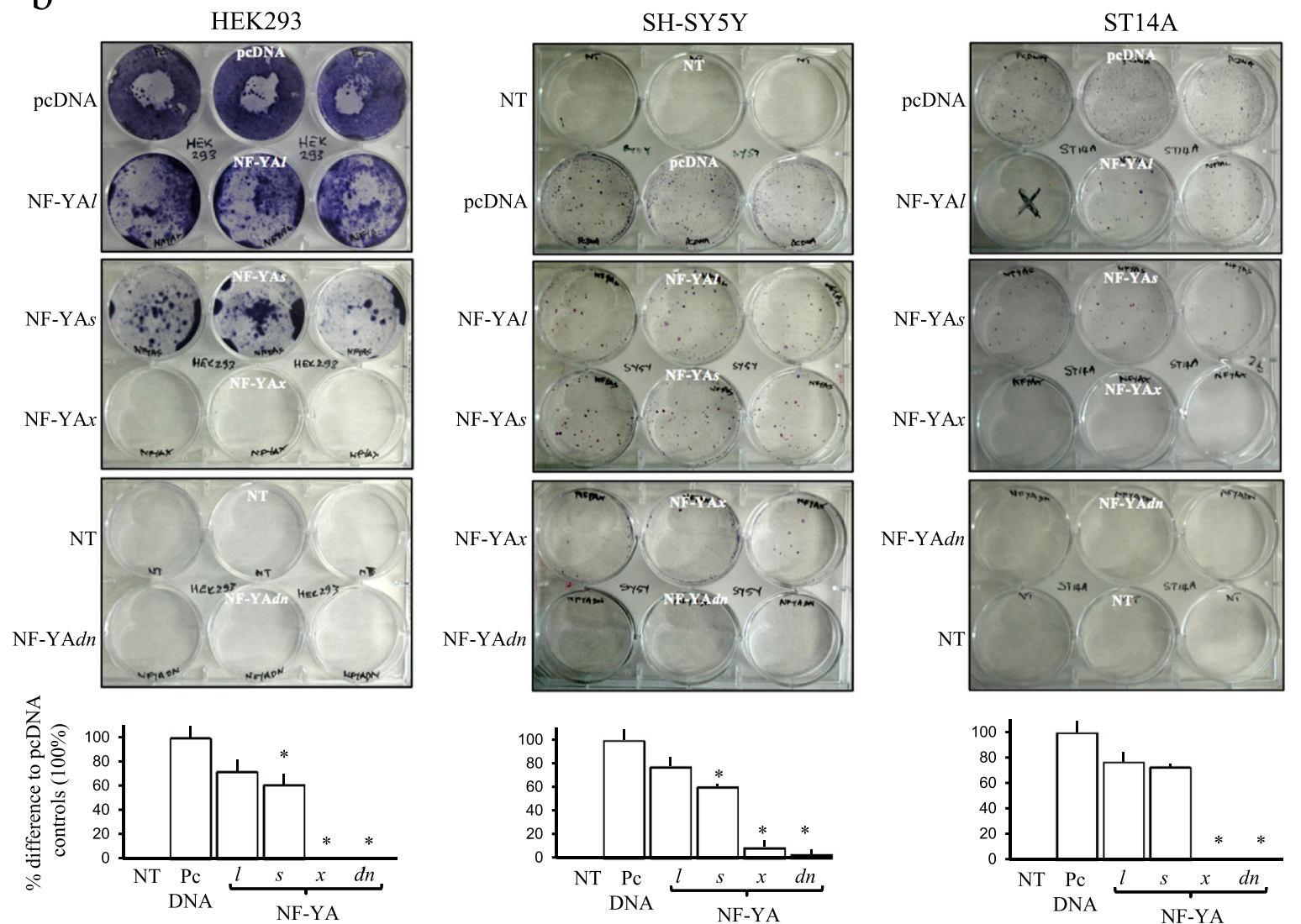

Fig. 5 (See legend on next page.) 
(See figure on previous page.)

Fig. 5 NF-YAx attenuates HEK-293, SH-SY5Y and ST14A colony formation. a Representative Micrographs (bar $=100 \mu m$ ) plus histograms, demonstrating Necrostatin-1 (Nec-1) $(100 \mu \mathrm{M})$ but not z-VAD-fmk (10 $\mu \mathrm{M})$ inhibition of NF-YAx-induced SH-SY5Y and HEK-293 cell-death. Histogram data are displayed as mean ( \pm SD) percent adherent/alive and suspension/dead cells, in three independent assays performed in duplicate $\left(^{*}=\right.$ significant). b Representative colony formation assays plus histograms, demonstrating inhibition of HEK293, SH-SY5Y and ST14A colony-formation by NF-YAx and NF-YAdn compared to NF-YAl, NF-YAs and pcDNA expression vectors. Histogram data are displayed as mean ( \pm SD) percentage difference to pcDNA-transfected controls, in three independent assays performed in triplicate $\left({ }^{*}=\right.$ significant)

In colony formation assays, sham-transfected SHSY5Y, HEK-293 and ST14A cells did not form colonies and pcDNA-transfected counterparts formed maximum colony numbers. Compared to respective pcDNA controls, in SH-SY5Y cells, NF-YAl-transfectants formed $22.6 \pm 7.5 \%$ fewer colonies $(p<0.001, n=9)$, NF-YAstransfectants $39.5 \pm 2.1 \%$ fewer colonies $(p<0.0001, \mathrm{n}=9)$, NF-YA $x$-transfectants $91.1 \pm 5.5 \%$ fewer colonies (means \pm SD) $(\mathrm{p}<0.0001, n=18)$ and NF-YAdn-transfectants did not form any colonies $(\mathrm{p}<0.0001, \mathrm{n}=18)$. In ST14A cells, NF-YA $l$ transfectants formed $23.3 \pm 7.5 \%$ fewer colonies $(P<0.0001, \mathrm{n}=9)$, NF-YAs $27.3 \pm 2.1 \%$ fewer colonies $(\mathrm{P}<0.001, \mathrm{n}=9)$ whereas NF-YAx and NF-YAdn-transfectants did not form colonies $(\mathrm{P}<0.001, \mathrm{n}=9$ for both). In HEK-293 cells, NF-YA $l$-transfectants formed $28 \pm 9.1 \%$ fewer colonies and NF-YAs-transfectants $38.6 \pm 8.5 \%$ fewer colonies $(\mathrm{p}<0.0001$ for both, $\mathrm{n}=9$ for each), whereas NF-YAx and NF-YAdn-transfectants did not form any colonies at all (Fig. 5b).

\section{NF-YAx induces Necroptosis}

NF-YAx-induced ST14A, SH-SY5Y and HEK293 celldeath was characterized by vacuolation, swelling and necrotic-lysis but not by apoptotic-body formation (Fig. 6a) or chromatin condensation (Fig. 4f). Western blots confirmed NF-YAx expression in dead suspension SH-SY5Y and HEK-293 cells (Fig. 6b) but did not detect caspase-3, caspase-9 or PARP-cleavage, enhanced JNK phosphorylation, reduced AIP/Alix expression or altered Bcl-xL, Bcl2, Mcl-1 and EglN3 expression (Fig. 6c and d). In both SH-SY5Y and HEK-293 cells, NF-YAx but not NF$\mathrm{YA} l$ reduced Bmil expression, induced KIF1B $\beta$ expression but did not alter EglN3 expression (Fig. 6c and d). In HEK293 cells, siRNA knockdown of NF-YA $x$-induced KIF1B $\beta$ expression significantly reduced NF-YA $x$-induced death from $24.6 \pm 0.75 \%(n=6)$ without siRNAs and $27.2 \pm 1.2 \%$ $(\mathrm{n}=6)$ with scrambled siRNAs to $5.4 \pm 0.61 \%(\mathrm{n}=6)$ with KIF1B-specific siRNAs (means \pm SD) $(p<0.0001$ versus siRNA controls) (Fig. 6e). Western blots also detected a significant $187.08 \pm 10.36 \%(n=3)$ increase in ratio of ubiquitinated proteins to $\beta$-actin and significant $148.2 \pm 8.6 \%(\mathrm{n}=$ 3 ) increase in the ratio p62 to $\beta$-actin (means \pm SD) ( $p \leq 0.036$ for all comparisons) in extracts from HEK-293 cells transfected with NF-YAx compared to pcDNA, NFYA $l$ or NF-YAs transfected counterparts (Fig. 6f).

\section{NF-YAx selects tumorigenic, doxorubicin-resistant cancer SCs}

Duplicate stable SH-SY5Y NF-YAx-transfectants were established and compared to stable control, NF-YA $l$ and NF-YAs-transfectants. Stable NF-YAl, NF-YAs, NF-YA $x 1$ and $x 2$-transfectants expressed similar levels of NF-YAl, NF-YAs and NF-YAx (Fig. 3c) and did not differ significantly in either proliferation or mitotic rates, assessed by MTS and thymidine-incorporation assays (Fig. 7a). NFYAl, NF-YAs and NF-YA $x$ stable transfectants all formed neuro-spheres in neural stem cell assays in vitro (Fig. 7b) and similar numbers of similar sized tumor spheroids in soft-agar tumorigenesis assays in vitro (Fig. 7c). In xenograft tumorigenesis assays in NSG mice, pcDNA, NF-YAs, NF-YA $x 1$ and $x 2$-transfectants formed sub-cutaneous tumours of similar dimensions, significantly larger than tumours formed by stable NF-YA $l$-transfectants $(\geq 2$ fold smaller, $p<0.049$ compared to all other stabletransfectants, $n=5$ per group) (Fig. $7 d$ ). In cell death assays, doxorubicin induced similar levels of stable pcDNA, NF-YA $l$ and NF-YAs-transfectant cell death at all concentrations at 6,24 and $48 \mathrm{~h}$. In contrast, stable NF-YA $x 1$ and $x 2$-transfectants exhibited significantly enhanced survival at 6 but not 24 or $48 \mathrm{~h}(\mathrm{p}<0.0001, \mathrm{n}=6$ for both) in the presence of $10 \mu \mathrm{M}$ doxorubicin, at 6 and 24 but not $48 \mathrm{~h}(\mathrm{p}<0.0001, \mathrm{n}=6$ for both $)$ in the presence of $5 \mu \mathrm{M}$ doxorubicin and at all time points in the presence of $0.01,0.1$ and $1 \mu \mathrm{M}$ doxorubicin $(\mathrm{p}<0.0001, \mathrm{n}=6$ for both at all time points and doses) (Fig. 7e).

In RT-PCR assays NF-YAx1 and $x 2$ transfectants exhibited higher levels of $\mathrm{p} 75^{\mathrm{NTR}}$, Nanog, Nestin and EgIN3 expression than NF-YAl and NF-YAs-transfectants, similar levels of Sox-2, CD133 and CD117 expression to NF-YAs-transfectants, elevated above control and NF-YAl-transfectants, high-level Bmi1 expression but did not express KIF1B $\beta$. In contrast, NF-YA $l$-transfectants expressed lower levels of $\mathrm{p} 75^{\mathrm{NTR}}$ and Nanog than the other transfectants. Bcl2, Mcl1, Bcl-xL, PUMA, BAD and Bax expression levels did not differ between stable transfectants (Fig. 8a, b and c). RNAs purified from xenograft tumours exhibited a similar pattern of CSC gene expression to corresponding cell cultures (Fig. 8d). PTC-209 (10 $\mu \mathrm{M}$ for $24 \mathrm{~h})$ abrogated proliferation of all stable transfectants (Fig. 9a) but did not reduce Bmi1 expression nor induce KIF1B $\beta$ mRNA expression in NFYA $x 1$ or $x 2$-transfectants (Fig. 9b). 


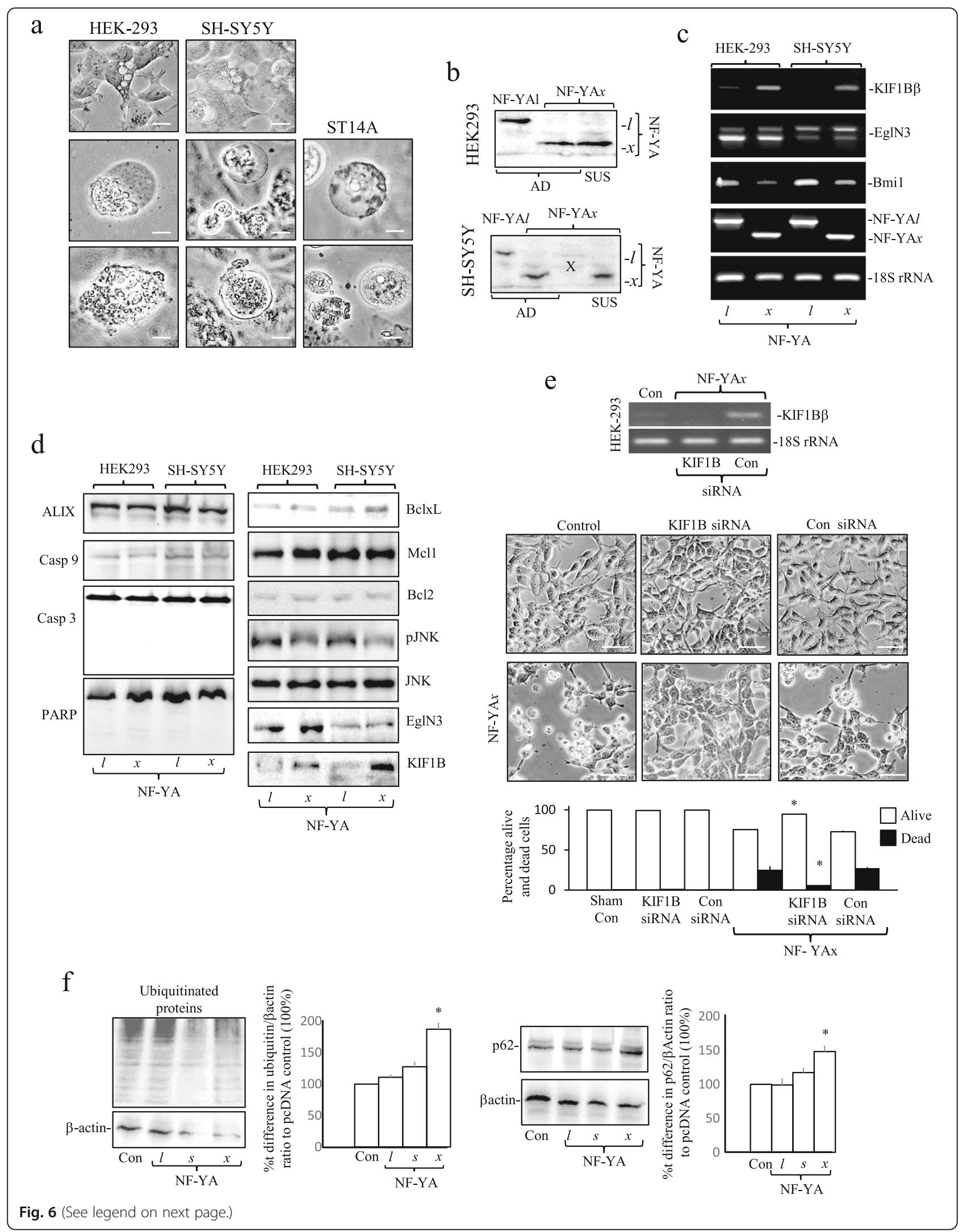


(See figure on previous page.)

Fig. 6 NF-YAx promotes KIF1Bß-dependent necroptosis. a Evidence of vacuolation, swelling and necrotic-lysis in HEK-293, SH-SY5Y and ST14A cells follwoing $48 \mathrm{~h}$ transfection with NF-YAx expression vector $(\mathrm{bar}=10 \mu \mathrm{m})$. b Western blots demonstrating the presence of both NF-YAl and NF-YAx in adherent (AD) and NF-YAx in dead/dying suspension (SUS) HEK-293 (upper panel) and SH-SY5Y (lower panel) extracts (20 $\mu \mathrm{g}$ ), following $48 \mathrm{~h}$ transfection. c RT-PCRs showing reduced Bmi-1 expression, induction of KIF1Bß expression but no change in EgIN3 expression in HEK-293 and SH-SY5Y cells, following $24 \mathrm{~h}$ transfection with NF-YAx but not NF-YAl expression vector, together with NF-YA isoform and $18 \mathrm{~S}$ rRNA levels. $\mathbf{d}$ Western blots showing stimulation of KIF1Bß expression but no changes in ALIX, BCIXL, Mcl1, BCl2, EgIN3, JNK, phosphorylated JNK levels and no cleavage of caspase 3 (Casp 3), caspase 9 (Casp 9) or PARP in HEK-293 and SH-SY5Y cells following 48 h transfection with NF-YAx but not NF-YAl expression vector $(30 \mu \mathrm{g}$ ). e RT-PCR demonstrating KIF1B-specific (KIF1B) but not scrambled siRNA (Con) knockdown of NF-YAx-induced KIF1BB expression in HEK-293 cells compared to 18 S RNA levels plus micrographs and a histogram showing prevention of NF-YAx-induced HEK-293 death by KIF1B-specific siRNAs (bottom 2nd panel) but not scrambled siRNAs (Con siRNA, bottom 3rd panel), compared to sham-transfected (bottom 1st panel) and siRNA-transfected controls (top 1st, 2nd and 3rd panels) (bar $=100 \mu \mathrm{m})$. Data are displayed as mean ( \pm SD) percent adherent vs suspension cells in two independent experiments performed in duplicate $\left({ }^{*}=\right.$ significant). $\mathbf{f}$ Western blots and histograms demonstrating significant increases in the ratio of ubiquitinated protein to $\beta$-actin in HEK-293 adherent plus suspension cell extracts (left panels and histogram) and ratio of p62 to $\beta$-actin in HEK-293 adherent plus suspension cell extracts (right panels and histogram), following $48 \mathrm{~h}$ transfection with NF-YAx compared to pcDNA, NF-YAl and NF-YAs expression vectors. Data are displayed as the mean ( \pm SD) densitometric ratio in three independent experiments ${ }^{*}=$ significant)

\section{Discussion}

We report a novel development and genotoxic stressregulated alternative splice mechanism for promoting embryonic neural-lineage progenitor and NB cell-death, characterized by a switch to alternative NF-YA splicing and expression of a novel cytotoxic NF-YAx extra shortform variant. This novel isoform, originally discovered in human primary stage 2 and stage 3 NB RNAs, was the exclusive NF-YA isoform expressed at a high-level in an advanced stage $3 \mathrm{NB}$ and was characterised as a novel NF-YA splice variant, exhibiting in-frame exon B, D and partial F skipping, responsible for truncating NF-YA transactivation domain sequence. NF-YAx readily competed with fully-spliced NF-YA $l$ in CCAAT-box binding NF-Y complex formation but in contrast to NF-YA $l$ and NF-YAs isoforms did not bind Sp1 and, therefore, represents a functional modifier of one of the more important physiological and cancer-associated transcription factors. In mouse embryos, NF-YAx expression coincided with the reported phase of neurotrophin-regulated KIF1B $\beta$-dependent sympathetic neuroblast-culling, unrestrained NF-YAx expression induced KIF1B $\beta$-dependent necroptosis in neurallineage progenitors and NB cells and association between doxorubicin-induced NF-YAx expression and necroptosis in NB cells, supports a pro-necroptotic cytotoxic function for NF-YA $x$ and a potential role in KIF1B $\beta$-dependent suppression of $\mathrm{NB}$ initiation, during development. On the other hand, propagation through selection of tumorigenic, doxorubicin-resistant CSC-like stable NF-YAx SH-SY5Y transfectants, resistant to NF-YAx cytotoxicity, not only helps to explain the high-level exclusive NF-YA $x$ expression detected in an advanced stage $3 \mathrm{NB}$ but also supports an additional potential role for NF-YAx, within the tumour context, in disease progression and identifies a potential mechanism for doxorubicin-induced post-therapeutic relapse, through CSC selection.

NF-YA $x$ cDNA was cloned from a stage 3 NB and sequence characterised as a novel NF-YA splice variant with in-frame exons B, D and partial $\mathrm{F}$ sequence skipping, adding to existing NF-YAl, NF-YAs and NF-YA L2-6 variants $[8,12,13,17]$, with potential implications for NB pathogenesis and progression. NF-YAx expression, detected in $20 \%$ (3/15) NF-YA-positive NB RNA samples, was the exclusive high-level NF-YA mRNA isoform expressed in a stage $3 \mathrm{NB}$ but was not detected in stage 1 and $4 \mathrm{NBs}$, human neonatal neural stem cells, HEK-293 human neurallineage embryonic kidney cells and 14 human NB cell lines. Although samples were not available for NF-YAx protein detection in primary NBs, expression of the endogenous NF-YAx protein was confirmed in SH-SY5Y cells, following treatment with doxorubicin, corroborating the characterisation of NF-YAx cDNA as a complete, inframe, non-mutated NF-YA splice variant that is readily translated into the NF-YAx protein in vitro and in vivo. Considering the relatively small number of NB samples analysed in this study, however, the possibility that NFYA $x$ mRNA expression is restricted to localized NB disseminated at most to local lymph nodes (Stages 2 and 3) [47] and may differentiate stage 3 from other disease stages, must await confirmation in a future larger NB cohort study.

NF-YAx mRNA was also expressed during mouse stage E12.5-E14.5 embryo development, consistent with identical exon organization of human (NCBI: NM 002505.5) and mouse (NCBI: NM_001110832.1) NF-YA genes. NF-YAx expression during stage E12.5-E14.5 embryo development coincides with the reported phase of neurotrophin-regulated EgIN3/KIF1B $\beta$-dependent sympathetic neuroblast-culling, required for sympathetic nervous system development and suppressing NB initiation [25-28, 48-55]. Furthermore, unrestrained NFYAx expression in neural-related progenitors and NB cells reduced expression of the KIF1B repressor Bmi1 [29], induced KIF1B $\beta$ expression, promoted KIF1B $\beta$ dependent necroptosis and abrogated colony forming capacity in vitro. This suggests that NF-YAx expression 


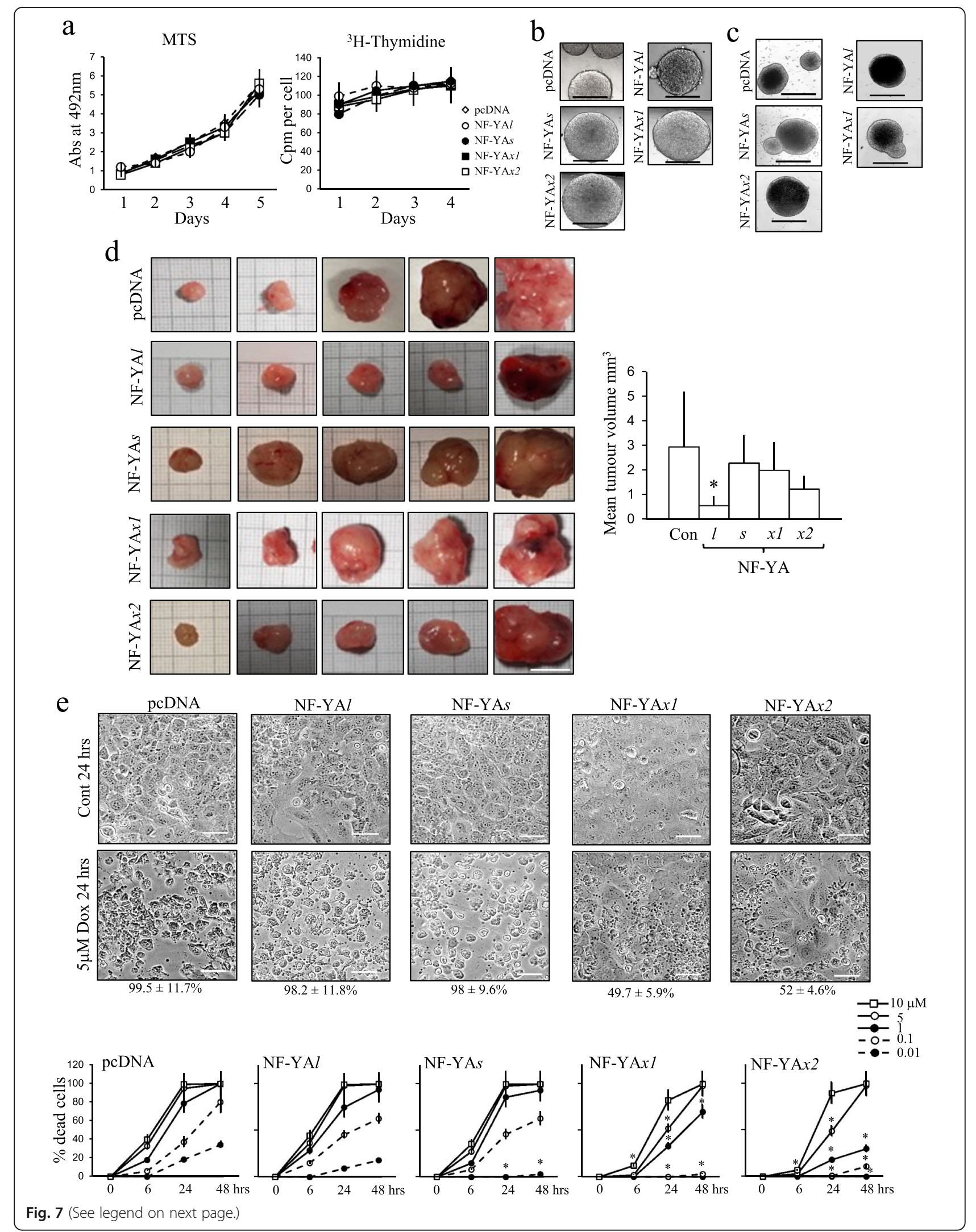




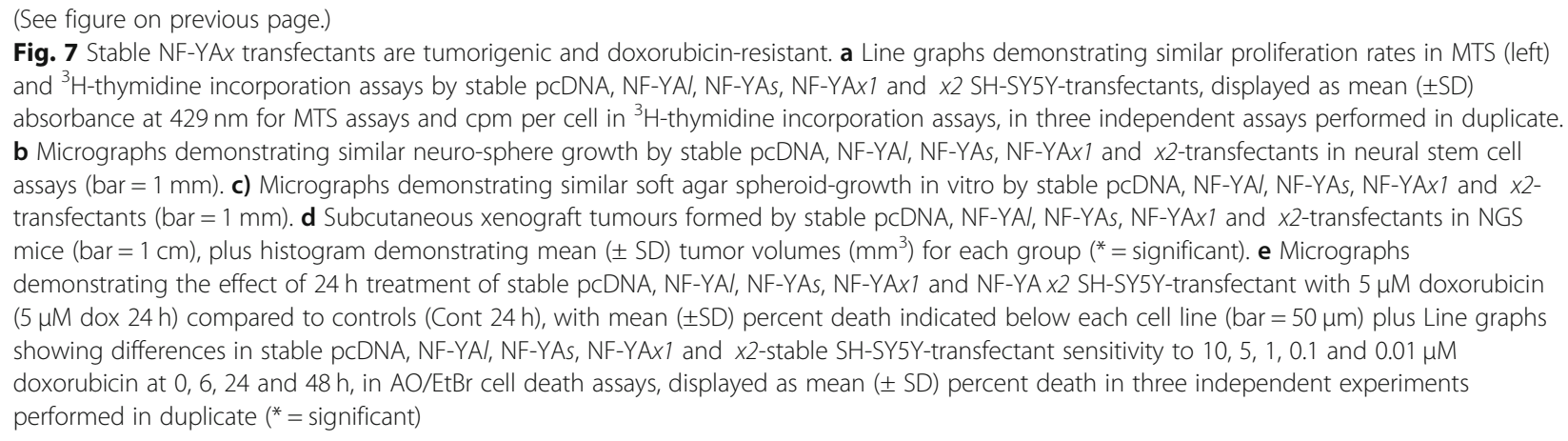

during mouse embryo development may be involved in KIF1B $\beta$-dependent sympathetic neuroblast culling and subsequent suppression of NB initiation. In contrast, unrestrained NF-YAx expression did not kill NB cells expressing TrkAIII oncoprotein [3]. This bears similarity to NGF-activated TrkA protection of sympathetic neuroblast against KIF1B $\beta$-dependent death $[26,27]$ and suggests that TrkAIII, which associates with $\mathrm{NB}$, may facilitate NB initiation by preventing KIF1B $\beta$-induced death in a manner analogous to $1 \mathrm{p} 36$-deletion of KIF1B, germline KIF1B mutations or Nmyc amplification [2532]. NF-YA $x$ induction of KIF1B $\beta$ expression, however, did not associate with changes in EglN3 expression, suggesting that this novel death-mechanism may act down stream of EglN3 through NF-YAx reduction of Bmil expression. Whether EglN3 promotion of alternative splicing [56], extends to NF-YAx remains to be determined.

NF-YA $x$ cytotoxicity to neural-related progenitors and NB cells was not associated with caspase 3, caspase 9 or PARP cleavage nor with changes in Bcl-2, BclXL, Mcl-1, NOXA, PUMA, BAX, BAD and AIP1/Alix expression or JNK-phosphorylation, was not inhibited by the pancaspase inhibitor $z$-VAD-fmk but was characterized by cell vacuolation, swelling and necrotic-lysis and inhibited by the RIPK-1 inhibitor necrostatin-1 [46], consistent with necroptosis rather than apoptosis $[57,58]$. NF-YAx also increased the levels of ubiquitinated and p62 proteins in HEK-293 cells, suggesting reduced autophagic flux, consistent with reports that both Bmil inhibition and KIF1B $\beta$ expression promote lysosomal/autophagosome uncoupling and necroptosis $[59,60]$, which may explain the NB suppressing function of $\operatorname{KIF} 1 B \beta[25,32$, $33,54,61,62]$. Although KIF1B $\beta$-induced apoptosis [32, $33,57,62,63]$ was not detected in this study, we do not exclude potential roles for KIF1B $\beta$-induced ROSmediated mitophagy or Drp1-dependent mitochondrialfission in this death mechanism [33, 60, 64-66].

NF-YA $x$ was significantly more cytotoxic to neuralrelated progenitor and NB cells than either NF-YA $l$ or NF-YAs, contrasting with a previous report that unrestrained NF-YA $l$ expression is highly cytotoxic and induces p53-dependent apoptosis [9]. This discrepancy can be explained by fact that p53 is compromised by SV40 large T-antigen in ST14A cells [40], by adenovirus- 5 in HEK-293 cells [41, 42] and by different mechanisms in SH-SY5Y cells [67-69], suggesting that this novel NF-YAx-dependent necroptosis mechanism may be restricted to genotoxic-stress under p53 compromised conditions. Furthermore, the sensitivity of ST14A neural progenitors, which exhibit low-level constitutive NF-YAx expression, to NF-YAx-induced necroptosis, confirms that this mechanism depends upon predominant NF-YAx expression. Unrestrained NF-YAdn expression, which contains a DNA bindingdomain mutation that prevents NF-Y binding and transcription [70], also induced HEK-293 and SHSY5Y necroptosis. This indicates that survival can switch to necroptosis in these cell types when either NF-Y binding is prevented (i.e. with NF-YAdn) or when a change in NF-Y function reaches a critical level (i.e. with NF-YAx ). Similar necroptotic-like neuronal death has also been reported during development $[25,48,54,62,71,72]$. A pro-necroptotic role for NF-YA $x$ was also supported by the association between doxorubicin-induced NF-YAx expression and SH-SY5Y cell-death, which was also characterised by vacuolation, swelling and cell lysis and significantly inhibited by the necroptosis inhibitor necrostatin-1 [46], consistent with a percentage of doxorubicininduced necroptosis. Confirmation of the role of NFYAX in this necroptotic proportion of doxorubicininduced death, however, must await development of reagents specific for NF-YAx depletion.

Doxorubicin induction of alternative NF-YAx splicing not only implicates the DNA damage-associated alternative splice mechanism [73] and a role for NFYAx in the response to genotoxic stress but also identifies NF-YA $x$ as a potential biomarker of response to genotoxic therapy, suggesting that NF-YAx expression in primary NBs could also reflect neo- 


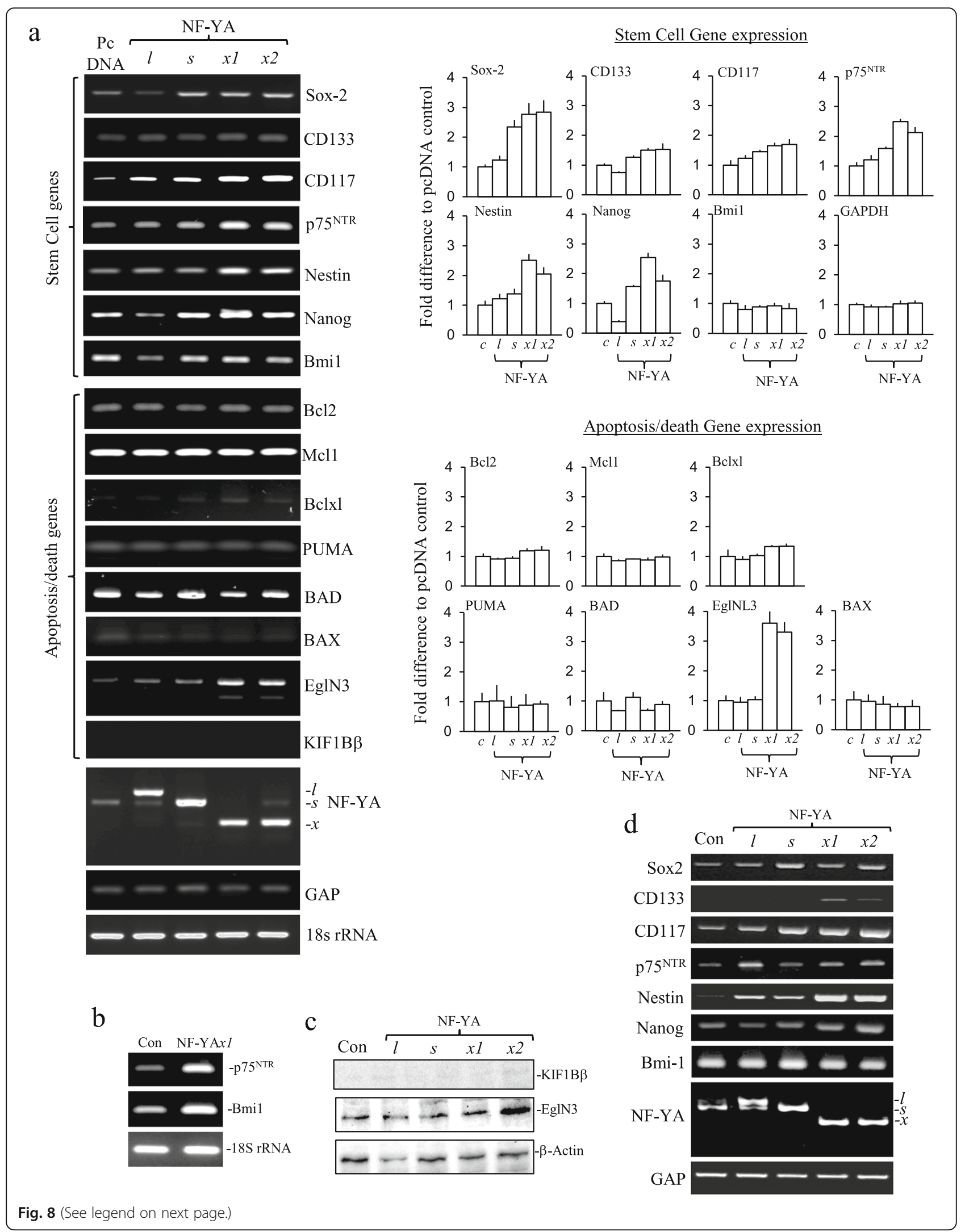


(See figure on previous page.)

Fig. 8 NF-YAx selects neuroblastoma CSCs. a Representative RT-PCRs and histograms demonstrating differences in stem cell-associated CD133, Sox-2, CD117, Nestin, Nanog, p75 ${ }^{\text {NTR }}$ and Bmi1 expression and apoptosis/related EgIN3, KIF1BR, BCl2, Mcl1, BClXL, PUMA, BAD and BAX expression plus NF-YAl, NF-YAs, NF-YAx, GAP and $18 \mathrm{~S}$ rRNA levels in stable pcDNA, NF-YAl, NF-YAs, NF-YAx1 and x2-transfectants. Histogram data are displayed as mean $( \pm \mathrm{SD}$ ) fold densitometric difference compared to pcDNA control transfectants, in three independent experiments performed in duplicate. b RT-PCR comparing p75 NTR, Bmi1 and $18 \mathrm{~S}$ rRNA levels in parental SH-SY5Y and stable NF-YAx1 transfected SH-SY5Y cells. c Western blots demonstrating EgIN3 and $\beta$-actin but not KIF1B $\beta$ expression in pcDNA, NF-YAl, NF-YAs, NF-YAx1 and x2-transfectant extracts (30 $\mu g$ ). d RTPCRs demonstrating Bmi1, p75 NTR , CD133, Sox-2, CD117, Nestin, Nanog, NF-YAl, NF-YAs, NF-YAx and GAP expression levels in selected xenograft tumours formed by pcDNA, NF-YAl, NF-YAs, NF-YAx1 and NF-YAx2-transfectants

adjuvant genotoxic chemotherapy [74]. Furthermore, the propagation of tumorigenic, doxorubicin-resistant, CSC-like stable NF-YAx SH-SY5Y transfectants, resistant to NF-YAx cytotoxicity, unveils an additional potential role for NF-YAx in genotoxic drug-induced, post-therapeutic relapse through CSC selection and maintenance. Moreover, the fact that NF-YAx expression was not induced by agents that promote ERstress, hypoxic-stress, differentiation or malignant behaviour, suggests that this alternative splice mechanism may be relatively restricted to conditions of genotoxic stress in NB cells.

NF-YA $x$ substitution of NF-YA $l$ in DNA-binding NF-Y complexes and the capacity of NF-YA $l$ and NFYAs but not NF-YAx to bind Sp1 (this study, [11, 44, $75]$ ), characterises NF-YAX as a potential modifier of both NF-Y and NF-Y-dependent Sp1 function. NFYA $x$ loss of $\mathrm{Sp} 1$ binding results from truncation of the aa 55-139 binding site for Sp1 in NF-YA $l$ and NF-YAs to aa 55-103 in NF-YAx (this study, [44, 76]). This truncation may also compromise NF-Y interaction with ZHX transcriptional repressors that bind NF-YA aa's 31140, with potential to also alter NF-Y/ZHX-regulated genes expression, including MDR-1 chemotherapeuticcytotoxicity-regulator and polo kinase-1 mitosis-regulator [77-81]. Transactivation-domain truncation may also weaken NF-Y function and interaction with other factors and the reduced size of NF-YAx, particularly in complexes with smaller NF-YB and NF-YC variants [82], may de-regulate transcription from promoters with precisely-spaced NF-Y-dependent transcriptional domains, e.g. ER-stress response gene promoters [83]. Although, NF-YAx-specific antibodies are not yet available for confirmation, by chromatin immunoprecipitation assay, that NF-YA $x$ is recruited to gene promoters in vivo, the fact that NF-YAx contains an intact DNA binding domain, competes with other isoforms in NF-Y complex formation and binds double stranded inverted CCAAT-box oligonucleotides in NF-Y complexed-form in vitro with similar kinetics to other NF-YA isoforms, strongly supports this probability. Although, we are only beginning to understand how NF-YAx may influence transcription, transient
NF-YAx expression reduced Bmi1 and induced KIF1B $\beta$ expression in neural-lineage progenitors and NB cells, and stable NF-YAx expression was associated with enhanced $\mathrm{p} 75^{\mathrm{NTR}}$, SOX2, Nestin, Nanog, CD117, CD133 and EglN3 expression.

Despite the acute cytotoxicity of NF-YAx to SH-SY5Y cells, stable SH-SY5Y transfectants exhibiting predominant NF-YAx expression were eventually propagated. These transfectants did not exhibit altered $\mathrm{Bcl} 2, \mathrm{Bcl}-\mathrm{xL}$ or $\mathrm{Mcl} 1$ expression nor constitutive KIF1B $\beta$ expression, suggesting selection based upon KIF1B $\beta$ repression but not Bcl2-regulated mitochondrial apoptosis. Stable NF-YAx transfectants also exhibited high constitutive Bmi1 expression, consistent with resistance to NF-YAx-induced KIF1B $\beta$-dependent cytotoxicity. This indicates that the SH-SY5Y cell line contains sub-populations that are sensitive and resistant to acute NF-YAx cytotoxicity, characterised by differences in NF-YAx regulation of Bmi1 and $\mathrm{KIF} 1 \mathrm{~B} \beta$ expression. The Bmi1 inhibitor PTC-209 [84], however, failed to induce KIF1B $\beta$ expression in stable NF-YA $x$-transfectants, indicating that Bmi1 alone is not responsible for KIF1B $\beta$ repression in the resistant subpopulations. PTC-209 did, however, abrogate the proliferation of all $\mathrm{SH}$ SY5Y-transfectants, consistent with the role of Bmi1 in proliferation and reports that PTC-209 induces G1/ $S$ checkpoint cell-cycle arrest [84-89]. We are currently investigating whether KIF1B repression in stable NF-YAx SH-SY5Y transfectants, as a potential selection mechanism, may involve inhibition EglN3 prolyl hydroxylase activity, required for KIF1B $\beta$ expression and regulated by oxygen and high/low-order complexing [55, 90-92] and/or changes in NB-associated NF-Y and Sp1-regulated microRNA expression [93-95].

Stable NF-YAx SH-SY5Y transfectants were more CSC-like than stable control, NF-YA $l$ or NF-YAs transfectants and were characterized by higher levels of p $75^{\text {NTR }}$, Nestin, Nanog and EglN3 expression compared to all other stable transfectants, similar levels of SOX2, CD133 and CD117 expression to NF-YAs-transfectants elevated over other transfectants, high-level Bmi1 expression and KIF1B $\beta$ repression, consistent with reports that elevated Bmi1 and EglN3 expression associated with 


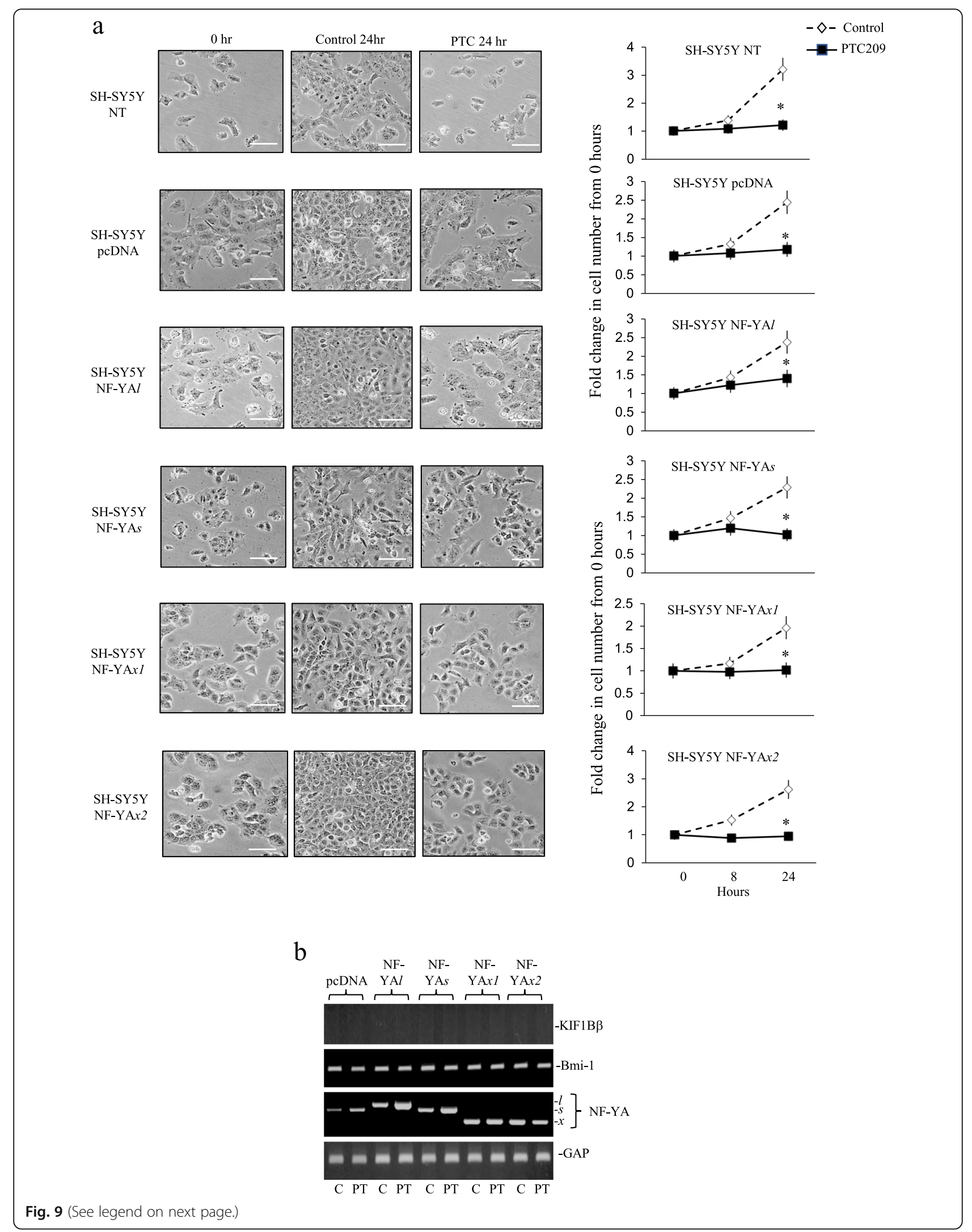


(See figure on previous page.)

Fig. 9 PTC-209 abrogates SH-SY5Y proliferation. a Phase contrast micrographs (bar $=100 \mu \mathrm{m}$ ) and histograms demonstrating abrogation of parental SH-SY5Y (NT-SH-SY5Y), stable NF-YAl, NF-YAs, NF-YAX1 and NF-YAx2 SH-SY5Y-transfectant proliferation, following $24 \mathrm{~h}$ treatment with $10 \mu \mathrm{M}$ PTC-209. Line graph data are displayed as mean ( \pm S.D.) fold change in cell number compared to 0-h controls, in three experiments performed in duplicate ${ }^{*}=$ significant). b RT-PCRs demonstrating no induction of KIF1Bß and no reduction in Bmi-1 expression in stable pcDNA, NF-YAl, NF-YAs, NF-YAx1 and NF-YAx2 SH-SY5Y-transfectants, following $24 \mathrm{~h}$ treatment with $10 \mu \mathrm{M}$ PTC-209

embryonic staminality [84-89, 92, 95, 96], elevated p75 ${ }^{\text {NTR }}$ expression characterises neural crest and $\mathrm{SH}$ SY5Y SCs [97-99] and KIF1B $\beta$-repression characterises undifferentiated aggressive NBs with $1 \mathrm{p} 36$ deletion or Nmyc amplification or that NBs that initiate in $K I F 1 B \beta^{-/-}$ mice $[25,26,29-33,100]$. Stable NF-YAx SH-SY5Y transfectants were tumorigenic both in vitro and in vivo, confirming that acute NF-YAx cytotoxicity selects a tumorigenic NB CSC-like subpopulations resistant to NF-YA $x$-induced KIF1B $\beta$-dependent necroptosis. This provides an explanation for high-level exclusive NFYA $x$ expression in the advanced stage $3 \mathrm{NB}$ and supports a role for NF-YAx, within the tumour context, in disease progression and suggests that doxorubicin induction of NF-YAx expression may also promote CSC selection in a potential mechanisms for posttherapeutic relapse, enhanced drug-resistance, increase adaptive plasticity and metastatic behaviour (this study, [101, 102]).

\section{Conclusions}

The discovery of NF-YAx mRNA in primary stage 2 and $3 \mathrm{NBs}$, its expression as the exclusive isoform in an advanced stage $3 \mathrm{NB}$, expression in stage E12.5 to E14.5 mouse embryos and induction by doxorubicin in NB cells, unveils a novel NF-YA splice mechanism and variant that is both regulated by and involved in NB, development and conditions of genotoxic-stress. NFYAx substitution of other isoforms in NF-Y complexes and loss of Sp1 binding capacity characterises this novel isoform as a functional modifier of one of the more important physiological and cancer-associated transcription factors. NF-YAx induction of KIF1B $\beta$ dependent embryonic neural-related progenitor and NB necroptosis, association with doxorubicin-induced necroptosis and expression in murine embryos at times corresponding to the phase of neurotrophinregulated KIF1B $\beta$-dependent sympathetic neuroblast culling, supports a predominant pro-necroptotic cytotoxic function for NF-YAx in neural progenitors and NB cells, with potential to suppress NB initiation during development. On the other hand, propagation by selection of tumorigenic, doxorubicin-resistant CSClike stable NF-YAx expressing SH-SY5Y transfectants, resistant to NF-YAx cytotoxicity, not only helps to explain the exclusive high-level NF-YAx expression in an advanced stage 3 primary NB but also suggests that NF-YA $x$ expression within the tumour context may promote disease progression and provides a possible doxorubicin-inducible mechanism for post-therapeutic relapse, through CSC selection and maintenance, with potential to enhance survival within stressful chemotherapeutic tumour microenvironments $[62,103]$.

\section{Abbreviations}

Bmi1: B cell-specific Moloney murine leukaemia virus integration site 1: CSC: cancer stem cell; KIF1B: kinesin family member 1B; NB: neuroblastoma; NF-YAl: long-form nuclear transcription factor YA; NF-YAs: short-form nuclear transcription factor YA; NF-YAX: extra short-form nuclear transcription factor YA; NSG mice: NOD severe combined immunodeficient gamma mice; Sp1: specificity protein 1

\section{Acknowledgements}

Lucia Cappabianca and Antonietta R. Farina are co-first authors. We wish to thank Professor R. Mantovani (University of Milan, Italy) for the NF-YAdn expression vector and Professor E. Cattaneo (University of Milan, Italy) for the ST14A cell line.

\section{Authors' contributions}

LC, ARF and ARM conceptualized, designed, supervised this study and take responsibility for data integrity and accuracy of analysis; LC, ARF, LD, PI, DD,

MS and ARM acquired, analysed, interpreted the data and provided materials and technical support; ARM wrote this manuscript; LC, ARF and ARM revised and edited the manuscript. All authors read and approved the final manuscript.

\section{Funding}

This work was funded by Fondazione Salvatore Maugeri (CTERZMAUGERI11) and AIRC grant (IG20801).

\section{Availability of data and materials}

The data sets used and/or analysed during this study are either included in this published article or are available from the corresponding author on reasonable request.

\section{Ethics approval and consent to participate}

Written consent was obtained for the use of all patient NB RNA samples by the tissue bank of the Italian Association of Haematology and Pediatric Oncology (Genova, Italy). Animals experiments were performed at University of Rome (La Sapienza) in accordance with Italian National and Rome university regulations.

\section{Consent for publication}

No identifying patient details are contained within this manuscript.

\section{Competing interests}

The authors declare that they have no competing interests.

\section{Author details}

${ }^{1}$ Department of Applied Clinical and Biotechnological Sciences, University of L'Aquila, Via Vetoio, Coppito 2, 67100 L'Aquila, Italy. ${ }^{2}$ Department of Molecular Medicine, La Sapienza University of Rome, 00161 Rome, Italy. ${ }^{3}$ Istituto Pasteur-Fondazione Cenci Bolognetti, La Sapienza University of Rome, 00161 Rome, Italy. ${ }^{4}$ Center for Life Nanoscience @ Sapienza, Istituto Italiano di Tecnologia, 00161 Rome, Italy. 
Received: 23 September 2019 Accepted: 13 November 2019 Published online: 05 December 2019

\section{References}

1. Modrek B, Lee C. A genomic view of alternative splicing. Nucleic Acids Res. 2001:30:13-9.

2. Kalnina Z, Zayakin P, Silina K, Line A. Alterations in pre-mRNA splicing in cancer. Genes Chromosomes Cancer. 2005;42:342-57.

3. Tacconelli A, Farina AR, Cappabianca L, DeSantis G, Tessitore A, Vetuschi A, et al. TrkA alternative splicing: a regulated tumor-promoting switch in human neuroblastoma. Cancer Cell. 2004;6:347-60.

4. Cappabianca L, Guadagni S, Maccarone R, Sebastiano M, Chiominto A, Farina AR, Mackay AR. A pilot study of alternative TrkAlll splicing in Merkel cell carcinoma: a potential oncogenic mechanism and novel therapeutic target. J Exp Clin Cancer Res. 2019:38:424. https://doi.org/10.1186/s13046-191425-3

5. Wang B-D, Lee NH. Aberrant splicing in Cancer and drug resistance. Cancers (Basel). 2018. https://doi.org/10.3390/cancers10110458.

6. Mantovani R. Nuclear factor $Y$ in development and disease. Biochim Biophys Acta Gene Regul Mech. 2017;1860:523-4.

7. Maity SN. NF-Y (CBF) regulation in specific cell types and mouse models. Biochim Biophys Acta Gene Regul Mech. 2017;1860:598-603.

8. Li G, Zhao H, Wang L, Wang Y, Guo X, Xu B. The animal nuclear factor Y: an enigmatic heterotrimeric transcription factor. Am J Cancer Res. 2018;8:110625.

9. Gurtner A, Manni I, Piaggio G. NF-Y in cancer: impact on cell transformation of a gene essential for proliferation. Biochim Biophys Acta Gene Regul Mech. 1860;2017:604-16.

10. Zambelli F, Pavesi G. Genome wide features, distribution and correlations of NF-Y binding sites. Biochim Biophys Acta Gene Regul Mech. 2017;1860:581-9.

11. Suske G. NF-Y and SP transcription factors-New insights in a long-standing liaison. Biochim Biophys Acta Gene Regul Mech. 2017;1860:590-7.

12. Maity SN, de Crombrugghe B. Biochemical analysis of the B subunit of the heterotrimeric CCAAT-binding factor. J Biol Chem. 1992;267:8286-92.

13. Coustry F, Maity SN, de Crombrugghe B. Studies on transcription activation by the multimeric CCAAT-binding factor CBF. J Biol Chem. 1995;270:468-75.

14. Romier C, Cocchiarella F, Mantovani R, Moras D. The NF-YB/NF-YC structure gives insight into DNA binding and transcription regulation by CAAT factor NF-Y. J Biol Chem. 2003;278:1336-45.

15. Li X-Y, Hooft van Huijsduijnen R, Mantovani R, Benoist C, Mathis D. Intronexon organisation of the NF-Y genes. J Biol Chem. 1992;267:8984-90.

16. Dolfini D, Gatta R, Mantovani R. NF-Y and the transcriptional activation of CCAAT promoters. Crit Rev Biochem Mol Biol. 2012;47:29-49.

17. Ge Y, Jensen TL, Matherly LH, Taub JW. Synergistic regulation of cystathionine-B-synthase-1b promoter by transcription factors NF-YA isoforms and Sp1. Biochim Biophys Acta. 2002;1579:73-80.

18. Zhu J, Zhang Y, Joe GJ, Pompetti R, Emerson SG. NF-YA activates multiple hematopoietic stem cell (HSC) regulatory genes and promotes HSC selfrenewal. Proc Natl Acad Sci U S A. 2005;102:11728-33.

19. Grskovic M, Chaivorapol C, Gaspar-Maia A, Li H, Ramalho-Santos M. Systemic identification of cis-regulatory sequences active in mouse and human embryonic stem cells. PLOS ONE. 2007; e145, doi:https://doi.org/10.1371/ jounal.pgen.0030145

20. Dolfini D, Minuzzo M, Pavesi G, Mantovani R. The short isoform of NF-YA belongs to the embryonic stem cell transcription factor circuitry. Stem Cells. 2012;30:2450-9.

21. Basile $V$, Baruffaldi $F$, Dolfini D, Belluti $S$, Benatti P, Ricci L, et al. NF-YA splice variants have different roles on muscle differentiation. Biochim Biophys Acta. 1859:2016:627-38.

22. Bevilacqua MA, Faniello MM, lovine B, Russo T, Cimino F, Costanzo F. Transcription factor NF-Y regulates differentiation of CaCo-2 cells. Arch Biochem Biophys. 2002;407:39-44.

23. Gu Z, Kuntz-Simon G, Rommelaere J, Cornelius J. Oncogenic transformationdependent expression of a transcription factor NF-Y subunit. Mol Carcinogen. 1999;24:294-9.

24. Ruggeri P, Farina AR, Cappabianca L, Di lanni N, Ragone M, Merolla S, et al, Neurotrophin and neurotrophin involvement in human neuroblastoma. In "Neuroblastoma" (ed: Hiroyuki Shimada, Intech, Rijeka, Cro) 2013: pp47-108.

25. Schlisio S, Kenchappa RS, Vredeveld LCW, George RE, Stewart R, Greulich H, et al. The kinesin KIF1BB acts downstream of EgIN3 to induce apoptosis and is a potential 1p36 tumor suppressor. Genes Dev. 2008;22:884-93.
26. Kristiansen M, Ham J. Programmed cell death during neuronal development: the sympathetic neuron model. Cell Death Diff. 2014;21:1025-35.

27. Fell SM, Li S, Wallis K, Kock A, Surova O, Rakili V, et al. Neuroblast differentiation during development and in neuroblastoma requires KIF1Bßmediated transport of TrkA. Genes Dev. 2017;31:1036-53.

28. Jiang M, Stanke J, Lahti JM. The connections between neural crest development and neuroblastoma. Curr Top Dev Biol. 2011;94:77-127.

29. Ochiai $H$, Takenobu H, Nakagawara A, Yamaguchi Y, Kimura M, Ohira M, et al. Bmi1 is a MYCN target gene that regulates tumorigenesis through repression of KIF1B 3 and TSLC1 in neuroblastoma. Oncogene. 2010;29:2681-90.

30. Ohira M, Kageyama H, Mihara M, Furuta S, Machida T, Shishikura T, et al. Identification and characterization of a 500-kb homozygous deleted region in a neuroblastoma cell line. Oncogene. 2000;19:4302-7.

31. Bauters $C$, Pigny $P$, Dahia PLM. A germline mutation of the KIF1B $\beta$ gene on 1 p36 in a family with neural and non-neural tumors. Hum Genet. 2008;124: 279-85.

32. Munirajan A, Ando K, Mukai A, Takahashi M, Suenaga Y, Ohira M, et al. KIF1B $\beta$ functions as a haploinsufficient tumor suppressor gene mapped to chromosome 1 p36.2 by inducing apoptotic cell death. J Biol Chem. 2008; 283:24426-34

33. Chen ZX, Wallis K, Fell SM, Sobrado VR, Hemmer MC, et al. RNA helicase a is a downstream mediator of KIF1B $\beta$ tumor-suppressor function in neuroblastoma. Cancer Discov. 2014;4:434-51.

34. Sharina IG, Martin E, Thomas A, Uray KL, Murad F. CCAAT-binding factor regulates expression of the beta-1 subunit of soluble guanylyl cyclase gene in the BE2 human neuroblastoma cell lines. Proc Natl Acad Sci U S A. 2003; 100:11523-8.

35. Boily $G$, Ouellet $S$, Langlois $S$, Lariviere $M$, Drouin $R$, Sinnett D. In vivo footprinting of the Glypican 3 (GPC3) promoter region in neuroblastoma cells. Biochim Biophys Acta. 2007;1769:182-93.

36. Yan YX, Zhao JX, Han S, Zhou NJ, Jia ZQ, Yao SJ, et al. Tetramethylpyrazine induces SH-SY5Y cell differentiation toward the neuronal phenotype through activation of the PI3K/Akt/Sp1/Topo11 $\beta$ pathway. Eur J Cell Biol. 2015;94:626-41.

37. Benfante R, Antonini RA, Vaccari M, Flora A, Chen F, Clementi F, et al. The expression of the human neuronal a3 $\mathrm{Na}+$, K+-ATPase subunit gene is regulated by the activity of the Sp1 and NF-Y transcription factors. Biochem J. 2005;386:63-72.

38. Cappabianca L, Farina AR, Tacconelli A, Mantovani R, Gulino A, Mackay AR. Reconstitution of TIMP-2 expression in SH-SY5Y neuroblastoma cells by 5 azacytidine is mediated transcriptionally by NF-Y through an inverted CCAAT site. Exp Cell Res. 2003:286:209-18.

39. Bernard J, Raguenez G, Kauffmann A, Valent A, Ripoche H, Joulin V, et al. MYCN-non-amplified metastatic neuroblastoma with good prognosis and regression: a molecular portrait of stage 4S. Mol Oncol. 2008;2:261-71.

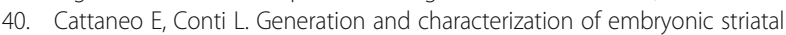
conditionally immortalized ST14A cells. J Neurosci Res. 1998;53:223-34.

41. Graham FL, Smiley J, Russel WC, Nairn R. Characteristics of a human cell line transformed by DNA from human adenovirus 5. J Gen Virol. 1977:36:59-74.

42. Stepanenko AA, Dmitrenko W. HEK293 in cell biology and cancer research: phenotype, karyotype, tumorigenicity, and stress-induced genomephenotype evolution. Gene. 2015;569:182-90.

43. Ruggeri P, Farina AR, Cappabianca L, Di lanni N, Ragone M, Merolla S, et al. The TrkAlll oncoprotein inhibits mitochondrial free radical ROS-induced death of SH-SY5Y neuroblastoma cells by augmenting SOD2 expression and activity at the mitochondria, within the context of a tumor stem cell-like phenotype. PLOS ONE. 2014;15:e94568.

44. Roder K, Wolf SS, Larkin KJ, Schweizer M. Interaction between the two ubiquitously expressed transcription factors NF-Y and Sp1. Gene. 1999:234:61-9.

45. Emami KH, Smale ST, Burke TW. Sp1 activation of TATA-less promoter requires a species-specific interaction involving transcription factor IID. Nucl Acids Res. 1998:26:839-46.

46. Degterev A, Hitomi J, Germscheid M, Ch'en IL, Korkina O, Teng X, et al. Identification of RIP1 kinase as a specific cellular target of necrostatins. Nat Chem Biol. 2008;4:313-21.

47. PDQ Pediatric Treatment Editorial Board. Neuroblastoma Treatment (PDQ $\left.{ }^{\circledast}\right)$ : Health Professional Version. 2018 Aug 17. In: PDQ Cancer Information Summaries [Internet]. Bethesda (MD): National Cancer Institute (US); 2002-. https://www.ncbi.nlm.nih.gov/books/NBK65747/.

48. Fernholm M. On the development of the sympathetic chain and the adrenal medulla in the mouse. Z Anat Entwicki Gesch. 1971;133:305-17. 
49. Mesner PW, Winters TR, Green SH. Nerve growth factor withdrawal-induced cell death in neuronal PC12 cells resembles that in sympathetic neurons. J Cell Biol. 1992;119:1669-80.

50. El Shamy WM, Linnarsson S, Lee K-F, Jaenisch R, Ernfors P. Prenatal and postnatal requirements for sympathetic neuroblast survival and innervation of specific targets. Development. 1996;122:491-500.

51. Pinon LGP, Minichiello L, Klein R, Davies AM. Timing of neuronal death in trkA, trkB and trkC mutant embryos reveals developmental changes in sensory neuron dependence on Trk sigaling. Development. 1996;122: 3255-61.

52. Lipscomb EA, Sarmiere PD, Crowder RJ, Freeman S. Expression of the SM-20 gene promotes death in nerve growth factor-dependent sympathetic neurons. J Neurochem. 1999:73:429-32.

53. Zhao C, Takita J, Tanaka Y, Setou M, Nakagawara T, Tekada S, et al. CharcotMarie-tooth disease type 2A caused by mutation in a microtubule motor KIF1Bbeta. Cell. 2001;105:587-97.

54. Sommer $L$, Rao M. Neural stem cells and regulation of cell number. Prog Neurobiol. 2002;66:1-18.

55. Lee $\mathrm{S}$, Nakamura $\mathrm{E}$, Yang H, Wei W, Linggi MS, Sajan MP, et al. Neurona apoptosis linked to EgIN3 prolyl hydroxylase and familial pheochromocytoma genes: developmental culling and cancer. Cancer Cell. 2005;8:155-67

56. Stoer A, Yang Y, Patel S, Evangelista AM, Aponte A, Wang G, et al. Prolyl hydroxylation regulates protein degradation, synthesis, and splicing in human induced pluripotent stem cell-derived cardiomyocytes. Cardiovasc Res. 2016;110:346-58.

57. Sperandio S, de Belle I, Bredesen DE. An alternative, nonapoptotic form of programmed cell death. Proc Natl Acad Sci U S A. 2000;97:14376-81.

58. Sperandio S, Poksay K, de Belle I, Lafuente MJ, Liu B, Nasir J, et al. Paraptosis: mediation by MAP kinases and inhibition by AIP-1/Alix. Cell Death Differ. 2004;11:1066-75.

59. Dey A, Mustafi SB, Saha S, Kumar Dhar Dwivedi S, Mukherjee P, Battacharya R. Inhibition of Bmi1 induces autophagy-mediated necroptosis. Autophagy. 2016;12:659-70.

60. Fricker M, Tolkovsky AM, Borutaite V, Coleman M, Brown GC. Neuronal cell death. Physiol Rev. 2018:98:813-80.

61. Schlisio S. Neuronal apoptosis by prolyl hydroxylation: implication in nervous system tumours and the Warburg conundrum. J Cell Mol Med. 2009:3:4104-12.

62. Nazio F, Bordi M, Cianfanelli V, Locatelli F, Cecconi F. Autophagy and cancer stem cells: molecular mechanisms and therapeutic applications. Cell Death Differ. 2019;26:690-702.

63. Hoa N, Myers MP, Douglass TG, Zhang JG, Delgado C, Driggers L, et al. Molecular mechanisms of paraptosis induction: implications for genetically modified tumor vaccine. PLoS One. 2009;4:e4631.

64. Blackstone C. KIF1B $\beta$ and neuroblastoma; failure to divide and cull. Dev Cell. 2016:36:127-8.

65. Angelina C, Tan ISY, Choo Z, Lee OZJ, Perviaz S, Chen ZX. KIF1Bß increases ROS to mediate apoptosis and reinforces its protein expression through $\mathrm{O} 2-$ in a positive feedback mechanism in neuroblastoma. Sci Reports. 2017;7:16867.

66. Han H, Chou C-C, Li R, Liu J, Zhang L, Zhu W, et al. Chalcomoracin is a potent anticancer agent acting through triggering oxidative stress via a mitophagy- and paraptosis-dependent mechanism. Sci Reports. 2018;8:9566. https://doi.org/10.1038/s41598-018-27724-3.

67. Toh WH, Logette E, Corcos L, Sabapathy K. Tap73 $\beta$ and DNp73 $\beta$ activate the expression of the prosurvival cappase-2s. Nuc Acids Res. 2006;36:4498-509.

68. Van Maerken T, Rihani A, Dreidax D, De Clercq S, Yigit N, Marine J-C, et al. Functional analysis of the p53 pathway in neuroblastoma cells using a small-molecule MDM2 antagonist nutlin-3. Mol Cancer Ther. 2011;10:983-93.

69. Miyazaki M, Otomo R, Matsushima-Hibiya Y, Suzuki H, Nakajima A, Abe N, et al. The p53 activator overcomes resistance to ALK inhibitors by regulating p53-target selectivity in ALK-driven neuroblastomas. Cell Death Disc. 2018;5: 56. https://doi.org/10.1038/s41420-018-0059-0

70. Mantovani R, Li XY, Pessara U, Hooft van Huisjduijnen R, Benoist C, Mathis D. Dominant negative analogues of NF-YA. J Biol Chem. 1994;269:20340-6.

71. Pilar G, Landmesser L. Ultrastructural differences during embryonic cell death in normal and peripherally deprived ciliary ganglia. J Cell Biol. 1976; 68:339-56.

72. Kutcher LM, Shaham S. Non-apoptotic cell death in animal development. Cell Death Differ. 2017;24:1326-36.
73. Chen J, Crutchley J, Zhang D, Owzar K, Kastan MB. Identification of a DNA damage-induced alternative splicing pathway that regulates p53 and cellular senescence markers. Cancer Disc. 2017;7:766-81.

74. Genesha VR, Schor NF. Pharmacological management of high-risk neuroblastoma in children. Paediatr Drugs. 2011;13:245-55.

75. Liang F, Schaufele F, Gardner DG. Functional interaction of NF-Y and Sp-1 is required for type a natriuretic peptide receptor gene transcription. J Biol Chem. 2001;276:1516-22

76. Yamada K, Tanaka T, Miyamoto K, Noguchi T. Sp family members and nuclear factor-Y cooperatively stimulate transcription from the rat pyruvate kinase $\mathrm{M}$ gene distal promoter region via their direct interactions. J Bio Chem. 2000;275:18129-37.

77. Wang Y, Wan C, Yu S, Yang L, Li B, Lu T, et al. Upregulating expression of NF-YC contributes to neuronal apoptosis via proapoptotic protein bim in rats' brain hippocampus following middle cerebral artery occlusion (MCAO). J Mol Neurosci. 2014;52:552-65.

78. Ma H, Yue X, Gao L, Liang X, Yan W, Zhang Z, et al. ZHX2 enhances the cytotoxicity of chemotherapeutic drugs in liver tumor cells by repressing MDR1 via interfering with NF-YA. Oncotarget. 2015:6:1049-63.

79. Kawata H, Yamada K, Shou Z, Mizutani T, Yazawa T, Yoshino M, et al. Zincfingers and homeoboxes (ZHX) 2, a novel member of the ZHX family functions as a transcription factor. Biochem J. 2003;373:747-57.

80. Hirano S, Yamada K, Kawata H, Shou Z, Mizutani T, Yazawa T, et al. Rat zincfingers and homeoboxes 1 (ZHX1), a nuclear factor-YA-interacting nuclear protein, forms a homodimer. Gene. 2002;290:107-14.

81. Yamada K, Osawa H, Granner DK. Identification of proteins that interact with NF-YA. FEBS Lett. 1999:460:41-5.

82. Ceribelli M, Benatti P, Imbriano C, Mantovani R. NF-YC complexity is generated by dual promoters and alternative splicing. J Biol Chem. 2009;284:34189-200.

83. Dolfini D, Zambelli F, Pedrazzoli M, Mantovani R, Pavesi G. A high definition look at the NF-Y regulome reveals genome-wide associations with selected transcription factors. Nucl Acid Res. 2016:44:4684-702.

84. Valk-Lingbeek ME, Bruggeman SWM, van Lohuizen M. Stem cells and cancer: the polycomb connection. Cell. 2004;118:409-18.

85. Molofsky AV, Pardal R, Iwashita T, Park IK, Clarke MF, Morrison SJ. Bmi-1 dependence distinguishes neural stem cell self-renewal from progenitor proliferation. Nature. 2003;425:962-7.

86. Molofsky AV, He S, Bydon M, Morrison SJ, Pardal R. Bmi-1 promotes neural stem cell self-renewal and neural development but not mouse growth and survival by repressing the p16Ink4a and p19Arf senescence pathways. Genes Dev. 2005;19:1432-7.

87. Fasano CA, Dimos JT, Ivanova NB, Lowry N, Lemischka IR, Temple S. shRNA knockdown of Bmi-1 reveals a critical role for p21-Rb pathway in NSC selfrenewal during development. Cell Stem Cell. 2007;1:87-99.

88. Lessard J, Baban S, Sauvageau G. Stage-specific expression of polycomb group genes in human bone marrow cells. Blood. 1998;91:1216-24.

89. Srinivasan M, Bharali DJ, Sudha T, Khedr M, Guest I, Sell S, et al. Downregulation of Bmi1 in breast cancer stem cells suppresses tumor growth and proliferation. Oncotarget. 2017:8:38731-42.

90. Nakayama K, Gazdoiu S, Abraham R, Pan ZQ, Ronai Z. Hypoxia-induced assembly of prolyly hydroxylase PHD3 into complexes: implications for its activity and susceptibility for degradation by the E3 ligase Siah2. Biochem J. 2007:401:217-26.

91. Zhou T, Erber L, Liu B, Gao Y, Ruan H-B, Chen Y. Proteomic analysis reveals diverse proline hydroxylation-mediated oxygen-sensing cellular pathways in cancer cells. Oncotarget. 2016;7:79154-69.

92. Appelhoff RJ, Tian YM, Raval RR, Turley H, Harris AL, Pugh CW, et al. Differential function of the prolyl hydroxylases PHD1, PHD2, and PHD3 in the regulation of hypoxia inducible factor. J Biol Chem. 2004;279:38458-65.

93. Mestdagh $P$, Bostrom A-K, Impens F, Fredlung E, Van Peer G, De Antonellis $P$, et al. The miR-17-92 microRNA cluster regulates multiple components of the TGFß-pathway in neuroblastoma. Mol Cell. 2010;40:762-73.

94. Ji M, Rao E, Ramachandrareddy H, Shen Y, Jiang C, Chen J, et al. The miR17-92 MicroRNA cluster is regulated by multiple mechanisms in B-cell malignancies. J Am Pathol. 2011;179:1645-56.

95. Narva E, Pursiheimo J-P, Laiho A, Rahkonen N, Emani MR, Viitala M, et al. Continuous hypoxic culturing of human embryonic stem cells enhances SSEA-3 and MYC levels. PLoS One. 2013;8:e78847.

96. Tuckerman JR, Zhao Y, Hewitson KS, Tian YM, Pugh CW, Ratcliff PJ, et al. Determination and comparison of specific activity of the HIF-prolyl hydroxylases. FEBS Lett. 2004;576:145-50. 
97. Achilles A, Trainor PA. Neural crest stem cells: discovery, properties and potential for therapy. Cell Res. 2012;22:288-304.

98. Biagiotti T, D'Amico M, Marzi I, Di Gennaro P, Arcangeli A, Wanke E, Oliviotto M. Cell renewing in neuroblastoma: electrophysiological and immunocytochemical characterization of stem cells and derivatives. Stem Cells. 2006;24:443-53.

99. Ross RA, Spengler BA. Human neuroblastoma stem cells. Semin Cancer Biol. 2007:17:241-7.

100. Li S, Fell SM, Surova O, Smedler E, Wallis K, Chen ZX, et al. The 1 p36 tumor suppressor KIF1B $\beta$ is required for calcineurin activation controlling mitochondrial fission and apoptosis. Dev Cell. 2016;36:164-78.

101. Pandian V, Ramraj S, Khan FH, Azim T, Aravindan N. Metastatic neuroblastoma cancer stem cells exhibit flexible plasticity and adaptive stemness signaling. Stem Cell Res Ther. 2015;6:2. https://doi.org/10.1186/ s13287-015-0002-8

102. Garner EF, Beierle EA. Cancer stem cells and their interaction with the tumour microenvironment in neuroblastoma. Cancers. 2016; 8:doi:https:// doi.org/10.3390/cancers8010005.

103. Zheng X, Naiditch J, Czurylo M, Jie C, Lautz T, Clark S, et al. Differential effect of long term drug selection with doxorubicin and virinostat on neuroblastoma cells with cancer cell characteristics. Cell Death Dis. 2013;4:e740.

\section{Publisher's Note}

Springer Nature remains neutral with regard to jurisdictional claims in published maps and institutional affiliations.

Ready to submit your research? Choose BMC and benefit from:

- fast, convenient online submission

- thorough peer review by experienced researchers in your field

- rapid publication on acceptance

- support for research data, including large and complex data types

- gold Open Access which fosters wider collaboration and increased citations

- maximum visibility for your research: over $100 \mathrm{M}$ website views per year

At $\mathrm{BMC}$, research is always in progress.

Learn more biomedcentral.com/submissions 